\title{
Mùi invariants and Milnor operations
}

\author{
MASAKI KAMEKO \\ MAMORU MiMURA
}

\begin{abstract}
We describe Mùi invariants in terms of Milnor operations and give a simple proof for Mùi's theorem on rings of invariants of polynomial tensor exterior algebras with respect to the action of finite general linear groups. Moreover, we compute some rings of invariants of Weyl groups of maximal non-toral elementary abelian $p$-subgroups of exceptional Lie groups.
\end{abstract}

$55 \mathrm{R} 40 ; 55 \mathrm{~S} 10$

\section{Introduction}

Let $p$ be a fixed odd prime, $q$ the power of $p$ and $\mathbb{F}_{q}$ the finite field of $q$ elements. Let

$$
P_{n}=\mathbb{F}_{q}\left[x_{1}, \ldots, x_{n}\right]
$$

be the polynomial algebra in $n$ variables $x_{1}, \ldots, x_{n}$ over the finite field $\mathbb{F}_{q}$. Let

$$
E_{n}^{r}=\Lambda^{r}\left(d x_{1}, \ldots, d x_{n}\right)
$$

be the $r^{\text {th }}$ component of the exterior algebra of $d x_{1}, \ldots, d x_{n}$ over the finite field $\mathbb{F}_{q}$ and let

$$
E_{n}=\bigoplus_{r=0}^{n} E_{n}^{r}
$$

be the exterior algebra of $d x_{1}, \ldots, d x_{n}$ over $\mathbb{F}_{q}$. Let

$$
P_{n} \otimes E_{n}
$$

be the polynomial tensor exterior algebra in $n$ variables $x_{1}, \ldots, x_{n}$ over the finite field $\mathbb{F}_{q}$. The general linear group $G L_{n}\left(\mathbb{F}_{q}\right)$ and the special linear group $S L_{n}\left(\mathbb{F}_{q}\right)$ act on both the polynomial algebra $P_{n}$ and the polynomial tensor exterior algebra $P_{n} \otimes E_{n}$. In [4], the ring of invariants of the polynomial algebra is determined by Dickson. In [7], Mùi determined the ring of invariants of the polynomial tensor exterior algebra and described the invariants in terms of determinants. 
In the first half of this paper, we describe the invariants in terms of Milnor operations and give a simpler proof for Mùi's theorem. With the notation in Section 2, we may state Mùi's theorems.

Theorem 1.1 (Mùi) The ring of invariants of the polynomial tensor exterior algebra $P_{n} \otimes E_{n}$ with respect to the action of the special linear group $S L_{n}\left(\mathbb{F}_{q}\right)$ is a free $P_{n}{ }^{S L_{n}\left(\mathbb{F}_{q}\right)}$-module with the basis $\left\{1, Q_{I} d x_{1} \ldots d x_{n}\right\}$, where $I$ ranges over $A_{n}^{\prime}$.

Theorem 1.2 (Mùi) The ring of invariants of the polynomial tensor exterior algebra $P_{n} \otimes E_{n}$ with respect to the action of the general linear group $G L_{n}\left(\mathbb{F}_{q}\right)$ is a free $P_{n}{ }^{G L_{n}\left(\mathbb{F}_{q}\right)}$-module with the basis $\left\{1, e_{n}^{q-2} Q_{I} d x_{1} \ldots d x_{n}\right\}$, where $I$ ranges over $A_{n}^{\prime}$.

The invariant $Q_{i_{1}} \ldots Q_{i_{n-r}} d x_{1} \ldots d x_{n}$ in Theorem 1.1 and Theorem 1.2 above is, up to sign, the same as the Mùi invariant $\left[r: i_{1}, \ldots, i_{n-r}\right]$ in [7]. The first half of this paper has some overlap with M C Crabb's work [3]. However, our point of view on Mùi invariants seems to be different from his.

The second half of this paper is a sequel to the authors' work in [6] on the invariant theory of Weyl groups of maximal non-toral elementary abelian $p$-subgroup $A$ of simply connected exceptional Lie groups. For $p$ odd prime, up to conjugation, there are only 6 of them, for $p=3, A=E_{F_{4}}^{3}, E_{3 E_{6}}^{4}, E_{2 E_{7}}^{4}, E_{E_{8}}^{5 a}, E_{E_{8}}^{5 b}$ and for $p=5, A=E_{E_{8}}^{3}$. They and their Weyl groups are described by Andersen et al [1]. We computed the polynomial part of the invariants of Weyl groups except for the case $p=3, A=E_{E_{8}}^{5 b}$ as described by the authors [6]. In this paper, we compute rings of invariants of polynomial tensor exterior algebras with respect to the action of Weyl groups except for the case $p=3, A=E_{E_{8}}^{5 b}$.

In Section 2, we set up the notation used in the above theorems. In Section 3, we prove Theorem 1.1 and Theorem 1.2. In Section 4, we state Theorem 4.2 and using this theorem, we compute rings of invariants of Weyl groups of maximal non-toral elementary abelian $p$-subgroups of simply connected exceptional Lie groups. In Section 5, we prepare for the proof of Theorem 4.1 and Theorem 4.2. In Section 6, we prove Theorem 4.1. In Section 7, we prove Theorem 4.2. In Appendix A, we prove that the invariant $Q_{i_{1}} \ldots Q_{i_{n-r}} d x_{1} \ldots d x_{n}$ in Theorem 1.1 and Theorem 1.2 above is, up to sign, equal to the Mùi invariant $\left[r: i_{1}, \ldots, i_{n-r}\right]$.

We thank N Yagita for informing us that a similar description of Mùi invariants to the above form is also known to him. 


\section{Preliminaries}

Let $K_{n}$ be the field of fractions of $P_{n}$. For a finite set $\left\{y_{1}, \ldots, y_{r}\right\}$, we denote by $\mathbb{F}_{q}\left\{y_{1}, \ldots, y_{r}\right\}$ the $\mathbb{F}_{q}$-vector space spanned by $\left\{y_{1}, \ldots, y_{r}\right\}$. Let $G L_{n}\left(\mathbb{F}_{q}\right)$ be the set of $n \times n$ invertible matrices with coefficients in $\mathbb{F}_{q}$. We denote by $M_{m, n}\left(\mathbb{F}_{q}\right)$ the set of $m \times n$ matrices with coefficients in $\mathbb{F}_{q}$. In this paper, we consider the contragredient action of the finite general linear group, that is, for $g \in G L_{n}\left(\mathbb{F}_{q}\right)$, we define the action of $g$ on $P_{n} \otimes E_{n}$ by

$$
g x_{i}=\sum_{j=1}^{n} a_{i, j}\left(g^{-1}\right) x_{j}, \quad g d x_{i}=\sum_{j=1}^{n} a_{i, j}\left(g^{-1}\right) d x_{j},
$$

for $i=1, \ldots, n$ and

$$
g(x \cdot y)=g(x) \cdot g(y),
$$

for $x, y$ in $P_{n} \otimes E_{n}$, where $a_{i, j}\left(g^{-1}\right)$ is the entry $(i, j)$ in the matrix $g^{-1}$. For $x_{i}, d x_{i}$ in the polynomial tensor exterior algebra $P_{n} \otimes E_{n}$, we define cohomological degrees of $x_{i}$, $d x_{i}$ by $\operatorname{deg} x_{i}=2, \operatorname{deg} d x_{i}=1$ for $i=1, \ldots, n$ and we consider $P_{n} \otimes E_{n}$ as a graded $\mathbb{F}_{q}$-algebra.

Now, we recall Milnor operations $Q_{j}$ for $j=0,1, \ldots$ The exterior algebra

$$
\Lambda\left(Q_{0}, Q_{1}, Q_{2}, \ldots\right)
$$

over $\mathbb{F}_{q}$, generated by Milnor operations, acts on the polynomial tensor exterior algebra $P_{n} \otimes E_{n}$ as follows; the Milnor operation $Q_{j}$ is a $P_{n}$-linear derivation

$$
Q_{j}: P_{n} \otimes E_{n}^{r} \rightarrow P_{n} \otimes E_{n}^{r-1}
$$

defined by the Cartan formula

$$
Q_{j}(x y)=\left(Q_{j} x\right) \cdot y+(-1)^{\operatorname{deg} x} x \cdot\left(Q_{j} y\right)
$$

for $x, y$ in $P_{n} \otimes E_{n}$ and the unstable conditions

$$
\begin{aligned}
Q_{j} d x_{i} & =x_{i}^{q^{j}}, \\
Q_{j} x_{i} & =0,
\end{aligned}
$$

for $i=1, \ldots, n, j \geq 0$. Thus, the action of Milnor operations $Q_{j}$ commutes with the action of the finite general linear group $G L_{n}\left(\mathbb{F}_{q}\right)$. 
It is also clear that the action of $Q_{j}$ on $P_{n}$ trivially extends to the quotient field $K_{n}$ and we may regard $Q_{j}$ as a $K_{n}$-linear homomorphism

$$
Q_{j}: K_{n} \otimes E_{n}^{r} \rightarrow K_{n} \otimes E_{n}^{r-1} .
$$

We set up additional notations for handling Milnor operations.

Definition 2.1 For a positive integer $n$, we denote by $S_{n}$ the set

$$
\{0,1, \ldots, n-1\} \text {. }
$$

Let $A_{n}$ be the set of subsets of $S_{n}$. We denote by $A_{n, r}$ the subset of $A_{n}$ consisting of

$$
I=\left\{i_{1}, \ldots, i_{r}\right\}
$$

such that

$$
0 \leq i_{1}<\cdots<i_{r}<n
$$

We write $Q_{I}$ for

$$
Q_{i_{1}} \ldots Q_{i_{r}} .
$$

We consider $A_{n, 0}$ as the set of empty set $\{\emptyset\}$ and define $Q_{\emptyset}$ to be 1 . It is also convenient for us to define $A_{n}^{\prime}$ to be the union of $A_{n, r}$, where $r$ ranges from 0 to $n-1$.

Definition 2.2 Let $I, J$ be elements of $A_{n}$. We define $\operatorname{sign}(I, J)$ as follows. If $I \cap J \neq \emptyset$, then $\operatorname{sign}(I, J)=0$. If $I \cap J=\emptyset$ and $I \cup J=\left\{k_{1}, \ldots, k_{r+s}\right\}$, then $\operatorname{sign}(I, J)$ is the sign of the permutation

$$
\left(\begin{array}{cccccc}
i_{1}, & \ldots, & i_{r}, & j_{1}, & \ldots, & j_{s} \\
k_{1}, & \ldots, & k_{r}, & k_{r+1}, & \ldots, & k_{r+s}
\end{array}\right),
$$

where $I=\left\{i_{1}, \ldots, i_{r}\right\}, J=\left\{j_{1}, \ldots, j_{s}\right\}, i_{1}<\cdots<i_{r}, j_{1}<\cdots<j_{s}$ and $k_{1}<\cdots<$ $k_{r+s}$.

The following proposition is immediate from the definition above.

Proposition 2.3 For $I, J$ in $A_{n}$, we have

$$
Q_{I} Q_{J}=\operatorname{sign}(I, J) Q_{K}=(-1)^{r s} \operatorname{sign}(J, I) Q_{K},
$$

where $K=I \cup J$. 
Finally, using Milnor operations in place of determinants, we describe Dickson invariants. We follow the notation of Wilkerson's paper [9]. Let $\Delta_{n}(X), f_{n}(X)$ be polynomials of $X$ over $P_{n}$ of homogeneous degree $q^{n}$ defined respectively by

$$
\begin{aligned}
\Delta_{n}(X) & =(-1)^{n} Q_{0} \ldots Q_{n} d x_{1} \ldots d x_{n} d X \\
& =\sum_{i=0}^{n}(-1)^{n-i}\left(Q_{0} \ldots \widehat{Q}_{i} \ldots Q_{n} d x_{1} \ldots d x_{n}\right) X^{q^{i}}, \\
f_{n}(X) & =\prod_{x \in \mathbb{F}_{q}\left\{x_{1}, \ldots, x_{n}\right\}}(X+x),
\end{aligned}
$$

where the cohomological degrees of $d X, X$ are 1,2 , and $Q_{i} d X=X^{q^{i}}, Q_{i} X=0$, respectively.

Proposition 2.4 The polynomial $\Delta_{n}(X)$ is divisible by the polynomial $f_{n}(X)$ and

$$
e_{n}\left(x_{1}, \ldots, x_{n}\right) f_{n}(X)=\Delta_{n}(X)
$$

where $e_{n}\left(x_{1}, \ldots, x_{n}\right)=Q_{0} \ldots Q_{n-1} d x_{1} \ldots d x_{n} \neq 0$.

Proof On the one hand, both $\Delta_{n}(X)$ and $f_{n}(X)$ have all $x \in \mathbb{F}_{q}\left\{x_{1}, \ldots, x_{n}\right\}$ as roots. On the other hand, the coefficient of $X^{q^{n}}$ in $\Delta_{n}(X)$ is

$$
e_{n}\left(x_{1}, \ldots, x_{n}\right)=Q_{0} \ldots Q_{n-1} d x_{1} \ldots d x_{n}
$$

and $f_{n}(X)$ is monic. Since both $\Delta_{n}(X)$ and $f_{n}(X)$ have the same homogeneous degree $q^{n}$ as polynomials of $X$, we have the required equality.

Thus, we have the following proposition.

Proposition 2.5 We may express $f_{n}(X)$ as follows:

$$
f_{n}(X)=\sum_{i=0}^{n}(-1)^{n-i} c_{n, i}\left(x_{1}, \ldots, x_{n}\right) X^{q^{i}}
$$

where

$$
Q_{0} \ldots \widehat{Q}_{i} \ldots Q_{n} d x_{1} \ldots d x_{n}=e_{n}\left(x_{1}, \ldots, x_{n}\right) c_{n, i}\left(x_{1}, \ldots, x_{n}\right)
$$

and $c_{n, n}\left(x_{1}, \ldots, x_{n}\right)=1$.

The above proposition defines Dickson invariants $c_{n, i}\left(x_{1}, \ldots, x_{n}\right)$ for $i=0, \ldots, n-1$. When it is convenient and if there is no risk of confusion, we write $e_{n}, c_{n, i}$ for $e_{n}\left(x_{1}, \ldots, x_{n}\right), c_{n, i}\left(x_{1}, \ldots, x_{n}\right)$, respectively. 
Proposition 2.6 There holds

$$
c_{n, 0}\left(x_{1}, \ldots, x_{n}\right)=e_{n}\left(x_{1}, \ldots, x_{n}\right)^{q-1} .
$$

Proof It is clear that

$$
\left(Q_{i_{1}} \ldots Q_{i_{n}} d x_{1} \ldots d x_{n}\right)^{q}=Q_{i_{1}+1} \ldots Q_{i_{n}+1} d x_{1} \ldots d x_{n}
$$

and so

$$
e_{n}\left(x_{1}, \ldots, x_{n}\right)^{q}=e_{n}\left(x_{1}, \ldots, x_{n}\right) c_{n, 0}\left(x_{1}, \ldots, x_{n}\right)
$$

From the above definitions of $c_{n, i}\left(x_{1}, \ldots, x_{n}\right)$ and $e_{n}\left(x_{1}, \ldots, x_{n}\right)$ and from the fact that, for $g \in G L_{n}\left(\mathbb{F}_{q}\right)$,

$$
g d x_{1} \ldots d x_{n}=\operatorname{det}\left(g^{-1}\right) d x_{1} \ldots d x_{n}
$$

it follows that

$$
g e_{n}\left(x_{1}, \ldots, x_{n}\right)=\operatorname{det}\left(g^{-1}\right) e_{n}\left(x_{1}, \ldots, x_{n}\right)
$$

and that

$$
g c_{n, i}\left(x_{1}, \ldots, x_{n}\right)=c_{n, i}\left(x_{1}, \ldots, x_{n}\right) .
$$

Thus, it is clear that $P_{n} S L_{n}\left(\mathbb{F}_{q}\right)$ contains $e_{n}$ and $c_{n, 1}, \ldots, c_{n, n-1}$ and that $P_{n}{ }^{G L_{n}\left(\mathbb{F}_{q}\right)}$ contains $c_{n, 0}, \ldots, c_{n, n-1}$. Indeed, the following results are well-known. For proofs, we refer the reader to Benson [2], Smith [8] and Wilkerson [9].

Theorem 2.7 (Dickson) The ring of invariants $P_{n} S L_{n}\left(\mathbb{F}_{q}\right)$ is a polynomial algebra generated by $c_{n, 1}, \ldots, c_{n, n-1}$ and $e_{n}$.

Theorem 2.8 (Dickson) The ring of invariants $P_{n} G L_{n}\left(\mathbb{F}_{q}\right)$ is a polynomial algebra generated by $c_{n, 0}, \ldots, c_{n, n-1}$.

In addition, we need the following proposition.

Proposition 2.9 Let

$$
\pi: \mathbb{F}_{q}\left[x_{1}, \ldots, x_{n}\right] \rightarrow \mathbb{F}_{q}\left[x_{1}, \ldots, x_{n-1}\right]
$$

be the obvious projection. Then, we have

$$
\pi\left(e_{n}\left(x_{1}, \ldots, x_{n}\right)\right)=0
$$

and, for $i=1, \ldots, n-1$,

$$
\pi\left(c_{n, i}\left(x_{1}, \ldots, x_{n}\right)\right)=c_{n-1, i-1}\left(x_{1}, \ldots, x_{n-1}\right)^{q} .
$$


Proof It is clear that $e_{n}\left(x_{1}, \ldots, x_{n}\right)$ is divisible by $x_{n}$, so we have

$$
\pi\left(e_{n}\left(x_{1}, \ldots, x_{n}\right)\right)=0 .
$$

On the one hand, we have

$$
\begin{aligned}
f_{n}(X) & =\prod_{\alpha \in \mathbb{F}_{q}} \prod_{x \in \mathbb{F}_{q}\left\{x_{1}, \ldots, x_{n-1}\right\}}\left(X+\alpha x_{n}+x\right) \\
& =\prod_{\alpha \in \mathbb{F}_{q}} f_{n-1}\left(X+\alpha x_{n}\right) \\
& =\prod_{\alpha \in \mathbb{F}_{q}}\left(f_{n-1}(X)+\alpha f_{n-1}\left(x_{n}\right)\right) \\
& =f_{n-1}(X)^{q}-f_{n-1}(X) f_{n-1}\left(x_{n}\right)^{q-1} .
\end{aligned}
$$

On the other hand, since $f_{n}\left(x_{n}\right)$ is divisible by $x_{n}$, we have

$$
\pi\left(f_{n}(X)\right)=f_{n-1}(X)^{q} .
$$

Comparing the coefficients of $X^{q^{i}}$, we have, for $i=1, \ldots, n-1$,

$$
\pi\left(c_{n, i}\left(x_{1}, \ldots, x_{n}\right)\right)=c_{n-1, i-1}\left(x_{1}, \ldots, x_{n-1}\right)^{q} .
$$

\section{Proof of Theorem 1.1 and Theorem 1.2}

In the case $n=1$, the invariants are obvious. In the case $r=0$, the invariants are calculated by Dickson. So, throughout the rest of this section, we assume $n \geq 2$ and $r>0$. To prove Theorem 1.1 and Theorem 1.2, it suffices to prove the following theorems.

Theorem 3.1 The submodule $\left(P_{n} \otimes E_{n}^{r}\right)^{S L_{n}\left(\mathbb{F}_{q}\right)}$ is a free $P_{n}{ }^{S L_{n}\left(\mathbb{F}_{q}\right)}$-module with the basis $\left\{Q_{I} d x_{1} \ldots d x_{n}\right\}$, where $I$ ranges over $A_{n, n-r}$.

Theorem 3.2 The submodule $\left(P_{n} \otimes E_{n}^{r}\right)^{G L_{n}\left(\mathbb{F}_{q}\right)}$ is a free $P_{n}{ }^{G L_{n}\left(\mathbb{F}_{q}\right)}$-module with the basis $\left\{e_{n}^{q-2} Q_{I} d x_{1} \ldots d x_{n}\right\}$, where $I$ ranges over $A_{n, n-r}$.

To begin with, we prove the following proposition.

Proposition 3.3 The elements $Q_{I} d x_{1} \ldots d x_{n}$ form a basis for $K_{n} \otimes E_{n}^{r}$, where $I$ ranges over $A_{n, n-r}$. 
Proof Firstly, we show the linear independence of $Q_{I} d x_{1} \ldots d x_{n}$. Suppose that

$$
a=\sum_{I \in A_{n, n-r}} a_{I} Q_{I} d x_{1} \ldots d x_{n}
$$

where $a_{I} \in K_{n}$. For each $I \in A_{n, n-r}$, let $J=S_{n} \backslash I$. It is clear that $J \cap I^{\prime} \neq \emptyset$ if $I^{\prime} \neq I$ in $A_{n, n-r}$. Hence, we have $\operatorname{sign}(J, I) \neq 0$ and $\operatorname{sign}\left(J, I^{\prime}\right)=0$ for $I^{\prime} \neq I \in A_{n, n-r}$. By Proposition 2.3, we have

$$
Q_{J} a=\operatorname{sign}(J, I) a_{I} Q_{0} \ldots Q_{n-1} d x_{1} \ldots d x_{n}=\operatorname{sign}(J, I) a_{I} e_{n} .
$$

Thus, if $a=0$, then $a_{I}=0$. Therefore, the terms $Q_{I} d x_{1} \ldots d x_{n}$ are linearly independent in $K_{n} \otimes E_{n}^{r}$.

On the other hand, since

$$
\operatorname{dim}_{K_{n}} K_{n} \otimes E_{n}^{r}=\left(\begin{array}{c}
n \\
r
\end{array}\right)
$$

is equal to the number of elements in $A_{n, n-r}$, we see, for dimensional reasons, that $Q_{I} d x_{1} \ldots d x_{n}$ 's form a basis for $K_{n} \otimes E_{n}^{r}$.

Lemma 3.4 Let $h_{I}$ be polynomials over $\mathbb{F}_{q}$ in $(n-1)$ variables, where $I$ ranges over $A_{n, n-1}$. Suppose that

$$
a_{0}=\sum_{I \in A_{n, n-1}} h_{I}\left(c_{n, n-1}, \ldots, c_{n, 1}\right) e_{n}{ }^{-1} Q_{I} d x_{1} \ldots d x_{n}
$$

is in $P_{n} \otimes E_{n}^{1}$. Then $h_{I}=0$ for each $I \in A_{n, n-1}$.

Proof of Theorem 3.1 Suppose that $a$ is an element in $P_{n} \otimes E_{n}^{r}$ and that $a$ is $S L_{n}\left(\mathbb{F}_{q}\right)-$ invariant. By Proposition 3.3, the elements $Q_{I} d x_{1} \ldots d x_{n}$ form a basis for $K_{n} \otimes E_{n}^{r}$. Hence, there exist $a_{I}$ in $K_{n}$ such that

$$
a=\sum_{I \in A_{n, n-r}} a_{I} Q_{I} d x_{1} \ldots d x_{n} .
$$

For $I \in A_{n, n-r}$, let $J=S_{n} \backslash I$. Then, $Q_{J} a$ is in $P_{n}$. As in the proof of Proposition 3.3, we have

$$
Q_{J} a=\operatorname{sign}(J, I) a_{I} e_{n} .
$$

Therefore, there are polynomials $f_{I, k}$ over $\mathbb{F}_{q}$ in $(n-1)$ variables such that

$$
a_{I}=\sum_{k \geq 0} f_{I, k}\left(c_{n, n-1}, \ldots, c_{n, 1}\right) e_{n}^{k-1} .
$$


Thus, we have

$$
a=\sum_{I \in A_{n, n-r}} \sum_{k \geq 0} f_{I, k}\left(c_{n, n-1}, \ldots, c_{n, 1}\right) e_{n}^{k-1} Q_{I} d x_{1} \ldots d x_{n} .
$$

It remains to show that $f_{I, 0}=0$ for each $I \in A_{n, n-r}$.

Let

$$
a_{0}=a-\sum_{I \in A_{n, n-r}} \sum_{k \geq 1} f_{I, k}\left(c_{n, n-1}, \ldots, c_{n, 1}\right) e_{n}^{k-1} Q_{I} d x_{1} \ldots d x_{n} .
$$

Then, we have that

$$
a_{0}=\sum_{I \in A_{n, n-r}} f_{I, 0}\left(c_{n, n-1}, \ldots, c_{n, 1}\right) e_{n}^{-1} Q_{I} d x_{1} \ldots d x_{n}
$$

and that $a_{0}$ is also in $P_{n} \otimes E_{n}^{r}$.

For $J \in A_{n, r-1}$, the element $Q_{J} a_{0}$ is in $P_{n} \otimes E_{n}^{1}$. By Proposition 2.3, we have

$$
Q_{J} a_{0}=\sum_{K \in A_{n, n-1}, I=K \backslash J, I \in A_{n, n-r}} \operatorname{sign}(J, I) f_{I, 0}\left(c_{n, n-1}, \ldots, c_{n, 1}\right) Q_{K} d x_{1} \ldots d x_{n} .
$$

Hence, by Lemma 3.4, we have $\operatorname{sign}(J, I) f_{I, 0}=0$. For each $I$ in $A_{n, n-r}$, there exists $J \in A_{n, r-1} \operatorname{such}$ that $\operatorname{sign}(J, I) \neq 0$. Therefore, we have $f_{I, 0}=0$ for each $I$. This completes the proof.

Proof of Theorem 3.2 As in the proof of Theorem 3.1, if $a \in P_{n} \otimes E_{n}^{r}$ is $G L_{n}\left(\mathbb{F}_{q}\right)-$ invariant, the element $a$ can be expressed in the form

$$
a=\sum_{I \in A_{n, n-r}} \sum_{k \geq 1} f_{I, k}\left(c_{n, n-1}, \ldots, c_{n, 1}\right) e_{n}^{k-1} Q_{I} d x_{1} \ldots d x_{n} .
$$

For $g \in G L_{n}\left(\mathbb{F}_{q}\right)$, we have

$$
g a=\sum_{I \in A_{n, n-r}} \sum_{k \geq 1} \operatorname{det}\left(g^{-1}\right)^{k} f_{I, k}\left(c_{n, n-1}, \ldots, c_{n, 1}\right) e_{n}^{k-1} Q_{I} d x_{1} \ldots d x_{n} .
$$

Therefore, $a$ is $G L_{n}\left(\mathbb{F}_{q}\right)$-invariant if and only if $f_{I, k}=0$ for $k \not \equiv 0$ modulo $q-1$. Hence, we have

$$
a=\sum_{I \in A_{n, n-r}} \sum_{m \geq 0} f_{I, m(q-1)+(q-1)}\left(c_{n, n-1}, \ldots, c_{n, 1}\right) e_{n}^{m(q-1)} e_{n}^{q-2} Q_{I} d x_{1} \ldots d x_{n} .
$$

Since $e_{n}^{q-1}=c_{n, 0}$, we may write

$$
a=\sum_{I \in A_{n, n-r}} f_{I}^{\prime}\left(c_{n, n-1}, \ldots, c_{n, 1}, c_{n, 0}\right) e_{n}^{q-2} Q_{I} d x_{1} \ldots d x_{n},
$$


where

$$
f_{I}^{\prime}\left(c_{n, n-1}, \ldots, c_{n, 0}\right)=\sum_{m \geq 0} f_{I, m(q-1)+(q-1)}\left(c_{n, n-1}, \ldots, c_{n, 1}\right) c_{n, 0}{ }^{m} .
$$

This completes the proof.

Proof of Lemma 3.4 For the sake of notational simplicity, let $I_{i}=S_{n} \backslash\{i\}$ and we write $h_{i}$ for $h_{I_{i}}$. Since $a$ is in $P_{n} \otimes E_{n}^{1}$, there are $\varphi_{1}, \ldots, \varphi_{n}$ in $P_{n}$ such that

$$
a_{0}=\varphi_{1} d x_{1}+\cdots+\varphi_{n} d x_{n}
$$

The coefficient $\varphi_{n}$ of $d x_{n}$ is given by

$$
\begin{aligned}
& \sum_{i=0}^{n-1} h_{i}\left(c_{n, n-1}, \ldots, c_{n, 1}\right) e_{n}^{-1} Q_{I_{i}} d x_{1} \ldots d x_{n-1} \\
= & \left\{\sum_{i=0}^{n-1} h_{i}\left(c_{n, n-1}, \ldots, c_{n, 1}\right) c_{n-1, i}\right\} e_{n}^{-1} e_{n-1} .
\end{aligned}
$$

Hence, we have

$$
\left\{\sum_{i=0}^{n-1} h_{i}\left(c_{n, n-1}, \ldots, c_{n, 1}\right) c_{n-1, i}\right\} e_{n-1}=e_{n} \varphi_{n} .
$$

By Proposition 2.9, the obvious projection

$$
\pi: \mathbb{F}_{q}\left[x_{1}, \ldots, x_{n}\right] \rightarrow \mathbb{F}_{q}\left[x_{1}, \ldots, x_{n-1}\right]
$$

maps $e_{n}, c_{n, i}$ to $0, c_{n-1, i-1}^{q}$, respectively. So, we have

$$
\sum_{i=0}^{n-1} h_{i}\left(c_{n-1, n-2}^{q}, \ldots, c_{n-1,0}^{q}\right) c_{n-1, i}=0 .
$$

Since $c_{n-1, i}(i=0, \ldots, n-2)$ are algebraically independent in $\mathbb{F}_{q}\left[x_{1}, \ldots, x_{n-1}\right]$ and since $c_{n-1, n-1}=1$, writing $y_{i}$ for $c_{n-1, i}$, we have the following equation:

$$
h_{n-1}\left(y_{n-2}^{q}, \ldots, y_{0}^{q}\right)+\sum_{i=0}^{n-2} h_{i}\left(y_{n-2}^{q}, \ldots, y_{0}^{q}\right) y_{i}=0 .
$$

Applying partial derivatives $\partial / \partial y_{i}$, we have

$$
h_{i}\left(y_{n-2}^{q}, \ldots, y_{0}^{q}\right)=0
$$


for $i=0, \ldots, n-2$. Hence, $h_{i}\left(y_{n-2}^{q}, \ldots, y_{0}^{q}\right)=0$ for $i=0, \ldots, n-2$. Substituting these to the previous equation (1), we also have $h_{n-1}\left(y_{n-2}^{q}, \ldots, y_{0}^{q}\right)=0$. Since we deal with polynomials over the finite field $\mathbb{F}_{q}$, we have

$$
h_{i}\left(y_{n-2}^{q}, \ldots, y_{0}^{q}\right)=h_{i}\left(y_{n-2}, \ldots, y_{0}\right)^{q}
$$

for $i=0, \ldots, n-1$. Therefore, we have $h_{i}\left(y_{n-2}, \ldots, y_{0}\right)=0$ for $i=0, \ldots, n-1$. Since $y_{0}, \ldots, y_{n-2}$ are algebraically independent, we have

$$
h_{i}=0
$$

as polynomials over $\mathbb{F}_{q}$ in $(n-1)$ variables for $i=0, \ldots, n-1$.

\section{Invariants of some Weyl groups}

In this section, we consider the invariant theory of polynomial tensor exterior algebras. In what follows, we assume that $n \geq 2$. To state Theorem 4.2, which is our main theorem on the invariant theory, we need some notation. Let

$$
P_{n-1}=\mathbb{F}_{q}\left[x_{2}, \ldots, x_{n}\right]
$$

be the subalgebra of $P_{n}$ generated by $x_{2}, \ldots, x_{n}$ and let $E_{n-1}$ be the subalgebra of $E_{n}$ generated by $d x_{2}, \ldots, d x_{n}$. Let $G_{1}$ be a subgroup of $S L_{n-1}\left(\mathbb{F}_{q}\right)$ which acts on $P_{n-1}$ and $P_{n-1} \otimes E_{n-1}$ both. Let $G$ be a subgroup of $S L_{n}\left(\mathbb{F}_{q}\right)$ consisting of the following matrices:

$$
\left(\begin{array}{l|l}
1 & m \\
\hline 0 & g_{1}
\end{array}\right),
$$

where $g_{1} \in G_{1}$ and $m \in M_{1, n-1}\left(\mathbb{F}_{q}\right)$. Obviously the group $G$ acts on $P_{n}$ and $P_{n} \otimes E_{n}$. Finally, let

$$
\mathcal{O}_{n-1}\left(x_{i}\right)=\prod_{x \in \mathbb{F}_{q}\left\{x_{2}, \ldots, x_{n}\right\}}\left(x_{i}+x\right)
$$

Theorem 4.1 Suppose that the ring of invariants $P_{n-1}^{G_{1}}$ is a polynomial algebra generated by homogeneous polynomials $f_{2}, \ldots, f_{n}$ in $(n-1)$ variables $x_{2}, \ldots, x_{n}$. Then, the ring of invariants $P_{n}^{G}$ is also a polynomial algebra generated by

$$
\mathcal{O}_{n-1}\left(x_{1}\right), f_{2}, \ldots, f_{n}
$$


This theorem is a particular case of a theorem of Kameko and Mimura [6, Theorem 2.5]. We use this theorem to compute the polynomial part of invariants $P_{n}^{G}$ which appear in our main theorem, Theorem 4.2. So, Theorem 4.2 below works effectively together with Theorem 4.1.

Theorem 4.2 Suppose that the ring of invariants $P_{n-1}^{G_{1}}$ is a polynomial algebra generated by homogeneous polynomials $f_{2}, \ldots, f_{n}$ in $(n-1)$ variables $x_{2}, \ldots, x_{n}$ and suppose that the ring of invariants $\left(P_{n-1} \otimes E_{n-1}\right)^{G_{1}}$ is a free $P_{n-1}^{G_{1}}$-module with a basis $\left\{v_{i}\right\}$, where $i=1, \ldots, 2^{n-1}$. Then, the ring of invariants $\left(P_{n} \otimes E_{n}\right)^{G}$ is a free $P_{n}^{G}$-module with the basis $\left\{v_{i}, Q_{I} d x_{1} \ldots d x_{n}\right\}$, where $i=1, \ldots, 2^{n-1}$ and $I$ ranges over $A_{n-1}$.

We prove Theorem 4.1 and Theorem 4.2 in Section 5, Section 6 and Section 7.

As an application of Theorem 4.1 and Theorem 4.2, we compute rings of invariants of the mod $-p$ cohomology of the classifying spaces of maximal non-toral elementary abelian $p$-subgroups of simply connected exceptional Lie groups with respect to the Weyl group action.

It is well-known that for an odd prime $p$, a simply connected exceptional Lie group $G$ does not have non-toral elementary abelian $p$-subgroups except for the cases $p=5$, $G=E_{8}$, and $p=3, G=F_{4}, E_{6}, E_{7}, E_{8}$ (see [1] and [5]). Andersen, Grodal, Møller and Viruel [1] described the Weyl groups of maximal non-toral elementary abelian $p$-subgroups as well as their action on the underlying elementary abelian $p$-subgroup explicitly for $p=3, G=E_{6}, E_{7}, E_{8}$. Up to conjugate, there are only 6 maximal non-toral elementary abelian $p$-subgroups of simply connected exceptional Lie groups. For $p=5, G=E_{8}$ and for $p=3, G=F_{4}, E_{6}, E_{7}$, there is one maximal non-toral elementary abelian $p$-subgroup for each $G$. We call them $E_{E_{8}}^{3}, E_{F_{4}}^{3}, E_{3 E_{6}}^{4}, E_{2 E_{7}}^{4}$, following the notation in [1]. For $p=3, G=E_{8}$, there are two maximal non-toral elementary abelian $p$-subgroups, say $E_{E_{8}}^{5 a}$ and $E_{E_{8}}^{5 b}$, where the superscript indicates the rank of elementary abelian $p$-subgroup. For a detailed account on non-toral elementary abelian $p$-subgroups, we refer the reader to Andersen et al [1, Section 8], and its references.

Let $A$ be an elementary abelian $p$-subgroup of a compact Lie group $G$. Suppose that $A$ is of rank $n$. We denote by $W(A)$ the Weyl group of $A$. Choosing a basis, say $\left\{a_{i}\right\}$, for $A$, we consider the Weyl group $W(A)$ as a subgroup of the finite general linear group $G L_{n}\left(\mathbb{F}_{p}\right)$. We write $H^{*} B A$ for the mod- $p$ cohomology of the classifying space $B A$. 
The Hurewicz homomorphism $h: A=\pi_{1}(B A) \rightarrow H_{1}\left(B A ; \mathbb{F}_{p}\right)$ is an isomorphism. We denote by $\left\{d t_{i}\right\}$ the dual basis of $\left\{h\left(a_{i}\right)\right\}$, so that $d t_{i}$ is the dual of $h\left(a_{i}\right)$ with respect to the basis $\left\{h\left(a_{i}\right)\right\}$ of $H_{1}\left(B A ; \mathbb{F}_{p}\right)$ for $i=1, \ldots, n$. Let $\beta: H^{1} B A \rightarrow H^{2} B A$ be the Bockstein homomorphism. Then, the mod $-p$ cohomology of $B A$ is a polynomial tensor exterior algebra

$$
H^{*} B A=\mathbb{F}_{p}\left[t_{1}, \ldots, t_{n}\right] \otimes \Lambda\left(d t_{1}, \ldots, d t_{n}\right),
$$

where $\operatorname{deg} t_{i}=2, \operatorname{deg} d t_{i}=1$ and $t_{i}=\beta\left(d t_{i}\right)$ for $i=1, \ldots, n$. We denote by $\Gamma H^{*} B A$ the polynomial part of $H^{*} B A$, that is,

$$
\Gamma H^{*} B A=\mathbb{F}_{p}\left[t_{1}, \ldots, t_{n}\right] .
$$

The action of the Weyl group $W(A)$ on $A=\pi_{1}(B A)$, given by

$$
g a_{i}=\sum_{j} a_{j, i}(g) a_{j},
$$

where $\left\{a_{i}\right\}$ is the fixed basis of $A$, induces the action of $W(A)$ on $H^{*} B A$, which is given by

$$
g t_{i}=\sum_{j} a_{i, j}\left(g^{-1}\right) t_{j}, \quad g d t_{i}=\sum_{j} a_{i, j}\left(g^{-1}\right) d t_{j},
$$

for $i=1, \ldots, n$.

Now, we compute

$$
\left(H^{*} B A\right)^{W(A)}
$$

for $A=E_{E_{8}}^{3}, E_{F_{4}}^{3}, E_{3 E_{6}}^{4}, E_{2 E_{7}}^{4}, E_{E_{8}}^{5 a}$ using Theorem 1.1, Theorem 4.1 and Theorem 4.2.

Proposition 4.3 For the above elementary abelian $p$-subgroup $A$, the ring of invariants $\left(H^{*} B A\right)^{W(A)}$ is given as follows:

(1) For $p=5, G=E_{8}, A=E_{E_{8}}^{3},\left(H^{*} B A\right)^{W(A)}$ is given by

$$
\mathbb{F}_{5}\left[x_{62}, x_{200}, x_{240}\right] \otimes \mathbb{F}_{5}\left\{1, Q_{I} u_{3}\right\},
$$

where $x_{62}=e_{3}\left(t_{1}, t_{2}, t_{3}\right), x_{200}=c_{3,2}\left(t_{1}, t_{2}, t_{3}\right), x_{240}=c_{3,1}\left(t_{1}, t_{2}, t_{3}\right), u_{3}=$ $d t_{1} d t_{2} d t_{3}$ and $I$ ranges over $A_{3}^{\prime}$;

(2) For $p=3, G=F_{4}, A=E_{F_{4}}^{3},\left(H^{*} B A\right)^{W(A)}$ is given by

$$
\mathbb{F}_{3}\left[x_{26}, x_{36}, x_{48}\right] \otimes \mathbb{F}_{3}\left\{1, Q_{I} u_{3}\right\},
$$

where $x_{26}=e_{3}\left(t_{1}, t_{2}, t_{3}\right), x_{36}=c_{3,2}\left(t_{1}, t_{2}, t_{3}\right), x_{48}=c_{3,1}\left(t_{1}, t_{2}, t_{3}\right), u_{3}=$ $d t_{1} d t_{2} d t_{3}$ and $I$ ranges over $A_{3}^{\prime}$; 
(3) For $p=3, G=E_{6}, A=E_{3 E_{6}}^{4},\left(H^{*} B A\right)^{W(A)}$ is given by

$$
\mathbb{F}_{3}\left[x_{26}, x_{36}, x_{48}, x_{54}\right] \otimes \mathbb{F}_{3}\left\{1, Q_{I} u_{3}, Q_{J} u_{4}\right\},
$$

where $x_{26}=e_{3}\left(t_{2}, t_{3}, t_{4}\right), x_{36}=c_{3,2}\left(t_{2}, t_{3}, t_{4}\right), x_{48}=c_{3,1}\left(t_{2}, t_{3}, t_{4}\right)$,

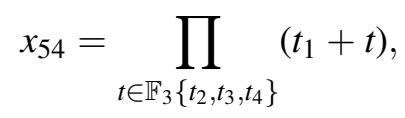

$u_{3}=d t_{2} d t_{3} d t_{4}, u_{4}=d t_{1} d t_{2} d t_{3} d t_{4}, I$ ranges over $A_{3}^{\prime}$ and $J$ ranges over $A_{3} ;$

(4) For $p=3, G=E_{7}, A=E_{2 E_{7}}^{4},\left(H^{*} B A\right)^{W(A)}$ is given by

$$
\mathbb{F}_{3}\left[x_{26}, x_{36}, x_{48}, x_{108}\right] \otimes \mathbb{F}_{3}\left\{1, Q_{I} u_{3}, x_{54} Q_{J} u_{4}\right\},
$$

where $x_{26}=e_{3}\left(t_{2}, t_{3}, t_{4}\right), x_{36}=c_{3,2}\left(t_{2}, t_{3}, t_{4}\right), x_{48}=c_{3,1}\left(t_{2}, t_{3}, t_{4}\right), x_{108}=x_{54}^{2}$,

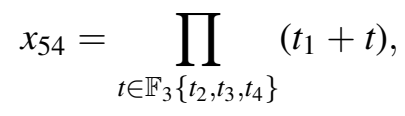

$u_{3}=d t_{2} d t_{3} d t_{4}, u_{4}=d t_{1} d t_{2} d t_{3} d t_{4}, I$ ranges over $A_{3}^{\prime}$ and $J$ ranges over $A_{3} ;$

(5) For $p=3, G=E_{8}, A=E_{E_{8}}^{5 a},\left(H^{*} B A\right)^{W(A)}$ is given by

$$
\mathbb{F}_{3}\left[x_{4}, x_{26}, x_{36}, x_{48}, x_{324}\right] \otimes \mathbb{F}_{3}\left\{1, Q_{I} u_{3}, x_{2} u_{1},\left(Q_{I} u_{3}\right) x_{2} u_{1}, x_{2} x_{162} Q_{J} u_{5}\right\},
$$

where $x_{4}=x_{2}^{2}, x_{26}=e_{3}\left(t_{2}, t_{3}, t_{4}\right), x_{36}=c_{3,2}\left(t_{2}, t_{3}, t_{4}\right), x_{48}=c_{3,1}\left(t_{2}, t_{3}, t_{4}\right)$, $x_{324}=x_{162}^{2}, x_{2}=t_{5}$,

$$
x_{162}=\prod_{t \in \mathbb{F}_{3}\left\{t_{2}, t_{3}, t_{4}, t_{5}\right\}}\left(t_{1}+t\right),
$$

$u_{1}=d t_{5}, u_{3}=d t_{2} d t_{3} d t_{4}, u_{5}=d t_{1} d t_{2} d t_{3} d t_{4} d t_{5}, I$ ranges over $A_{3}^{\prime}$ and $J$ ranges over $A_{4}$,

where the subscripts of $u$ and $x$ indicate their cohomological degrees.

Proof (1), (2) In the case $p=5, G=E_{8}, A=E_{E_{8}}^{3}$ and in the case $p=3, G=F_{4}$, $A=E_{F_{4}}^{3}$, the Weyl group is $S L_{3}\left(\mathbb{F}_{p}\right)$. Therefore, it is the case of Mùi invariants and it is immediate from Theorem 1.1.

(3) In the case $p=3, G=E_{6}, A=E_{E_{6}}^{4}$, the Weyl group $W(A)$ is the subgroup of $S L_{4}\left(\mathbb{F}_{3}\right)$ consisting of the following matrices:

$$
\left(\begin{array}{l|l}
1 & m \\
\hline 0 & g_{1}
\end{array}\right),
$$


where $m \in M_{1,3}\left(\mathbb{F}_{3}\right), g_{1} \in S L_{3}\left(\mathbb{F}_{3}\right)$. The result is immediate from Theorem 1.1, Theorem 4.1 and Theorem 4.2.

(4) In the case $p=3, G=E_{7}$, the Weyl group $W(A)$ is the subgroup of $G L_{4}\left(\mathbb{F}_{3}\right)$ consisting of the following matrices:

$$
\left(\begin{array}{c|c}
\varepsilon_{1} & m \\
\hline 0 & g_{1}
\end{array}\right),
$$

where $\varepsilon_{1} \in \mathbb{F}_{3}^{\times}=\{1,2\}, m \in M_{1,3}\left(\mathbb{F}_{3}\right), g_{1} \in S L_{3}\left(\mathbb{F}_{3}\right)$. Firstly, we compute the ring of invariants of a subgroup $W_{0}$ of $W(A)$. The subgroup $W_{0}$ is the subgroup of $W(A)$ consisting of the matrices

$$
\left(\begin{array}{l|l}
1 & m \\
\hline 0 & g_{1}
\end{array}\right),
$$

where $m \in M_{1,3}\left(\mathbb{F}_{3}\right), g_{1} \in S L_{3}\left(\mathbb{F}_{3}\right)$. By Theorem 1.1, Theorem 4.1 and Theorem 4.2, we have

$$
\left(H^{*} B A\right)^{W_{0}}=\mathbb{F}_{3}\left[x_{26}, x_{36}, x_{48}, x_{54}\right] \otimes \mathbb{F}_{3}\left\{1, Q_{I} u_{3}, Q_{J} u_{4}\right\},
$$

where $I$ ranges over $A_{3}^{\prime}$ and $J$ ranges over $A_{3}$. Let

$$
R=\mathbb{F}_{3}\left[x_{26}, x_{36}, x_{48}, x_{108}\right],
$$

and let

$$
M=\mathbb{F}_{3}\left\{x_{54}^{\delta}, x_{54}^{\delta} Q_{I} u_{3}, x_{54}^{\delta} Q_{J} u_{4}\right\},
$$

where $x_{108}=x_{54}^{2}, \delta \in\{0,1\}, I$ ranges over $A_{3}^{\prime}$ and $J$ ranges over $A_{3}$. Then, we have

$$
\left(H^{*} B A\right)^{W_{0}}=R \otimes M .
$$

Next, we calculate the ring of invariants $\left(H^{*} B A\right)^{W(A)}$ as a subspace of $\left(H^{*} B A\right)^{W_{0}}$. Put

$$
\alpha=\left(\begin{array}{c|ccc}
2 & 0 & 0 & 0 \\
\hline 0 & 1 & 0 & 0 \\
0 & 0 & 1 & 0 \\
0 & 0 & 0 & 1
\end{array}\right) .
$$

Then, for $x \in R$, we have $\alpha x=x$ and we also have the following direct sum decomposition:

$$
M=M_{1} \oplus M_{2},
$$

where $M_{i}=\{x \in M \mid \alpha x=i x\}$ for $i=1$, 2. In particular, we have

$$
M_{1}=\mathbb{F}_{3}\left\{1, Q_{I} u_{3}, x_{54} Q_{J} u_{4}\right\} .
$$


Since the Weyl group $W(A)$ is generated by $W_{0}$ and $\alpha$, an element $x$ in $R \otimes M$ is $W(A)$-invariant if and only if $\alpha x=x$. Hence, we have

$$
\left(H^{*} B A\right)^{W(A)}=R \otimes M_{1} .
$$

(5) In the case $p=3, G=E_{8}, A=E_{E_{8}}^{5 a}$, the Weyl group $W(A)$ is the subgroup of $G L_{5}\left(\mathbb{F}_{3}\right)$ consisting of the following matrices:

$$
\left(\begin{array}{c|c|c}
\varepsilon_{1} & m_{0} & m_{1} \\
\hline 0 & g_{1} & 0 \\
\hline 0 & 0 & \varepsilon_{2}
\end{array}\right),
$$

where $\varepsilon_{1}, \varepsilon_{2} \in \mathbb{F}_{3}^{\times}=\{1,2\}, m_{0} \in M_{1,3}\left(\mathbb{F}_{3}\right), m_{1} \in M_{1,1}\left(\mathbb{F}_{3}\right), g_{1} \in S L_{3}\left(\mathbb{F}_{3}\right)$. We consider the subgroup $W_{0}$ of $W(A)$ consisting of the following matrices:

$$
\left(\begin{array}{c|c|c}
1 & m_{0} & m_{1} \\
\hline 0 & g_{1} & 0 \\
\hline 0 & 0 & 1
\end{array}\right),
$$

where $g_{1} \in S L_{3}\left(\mathbb{F}_{3}\right), m_{0} \in M_{1,3}\left(\mathbb{F}_{3}\right), m_{1} \in M_{1,1}\left(\mathbb{F}_{3}\right)$. By Theorem 4.1 and Theorem 4.2, we have

$$
\left(H^{*} B A\right)^{W_{0}}=\mathbb{F}_{3}\left[x_{2}, x_{26}, x_{36}, x_{48}, x_{162}\right] \otimes \mathbb{F}_{3}\left\{1, Q_{I} u_{3}, u_{1},\left(Q_{I} u_{3}\right) u_{1}, Q_{J} u_{5}\right\},
$$

where $I$ ranges over $A_{3}^{\prime}$ and $J$ ranges over $A_{4}$. Let

$$
R=\mathbb{F}_{3}\left[x_{4}, x_{26}, x_{36}, x_{48}, x_{324}\right]
$$

and let

$$
M=\mathbb{F}_{3}\left\{x_{2}^{\delta_{1}} x_{162}^{\delta_{2}}, x_{2}^{\delta_{1}} x_{162}^{\delta_{2}} Q_{I} u_{3}, x_{2}^{\delta_{1}} x_{162}^{\delta_{2}} u_{1}, x_{2}^{\delta_{1}} x_{162}^{\delta_{2}}\left(Q_{I} u_{3}\right) u_{1}, x_{2}^{\delta_{1}} x_{162}^{\delta_{2}} Q_{J} u_{5}\right\},
$$

where $\delta_{1}, \delta_{2} \in\{0,1\}, I$ ranges over $A_{3}^{\prime}$ and $J$ ranges over $A_{4}$. Consider matrices

$$
\alpha=\left(\begin{array}{c|ccc|c}
2 & 0 & 0 & 0 & 0 \\
\hline 0 & 1 & 0 & 0 & 0 \\
0 & 0 & 1 & 0 & 0 \\
0 & 0 & 0 & 1 & 0 \\
\hline 0 & 0 & 0 & 0 & 1
\end{array}\right), \beta=\left(\begin{array}{c|ccc|c}
1 & 0 & 0 & 0 & 0 \\
\hline 0 & 1 & 0 & 0 & 0 \\
0 & 0 & 1 & 0 & 0 \\
0 & 0 & 0 & 1 & 0 \\
\hline 0 & 0 & 0 & 0 & 2
\end{array}\right) .
$$

Then, we have $\alpha x=x$ and $\beta x=x$ for $x \in R$. Furthermore, it is also clear that we have the following direct sum decomposition:

$$
M=M_{1,1} \oplus M_{1,2} \oplus M_{2,1} \oplus M_{2,2},
$$


where

$$
M_{i, j}=\{x \in M \mid \alpha x=i x, \beta x=j x\} .
$$

In particular, we have

$$
M_{1,1}=\mathbb{F}_{3}\left\{1, x_{2} u_{1}, Q_{I} u_{3}, x_{2}\left(Q_{I} u_{3}\right) u_{1}, x_{2} x_{162} Q_{J} u_{5}\right\} .
$$

Since $W(A)$ is generated by $W_{0}$ and $\alpha, \beta$ in the above, $x \in\left(H^{*} B A\right)^{W_{0}}$ is $W(A)$-invariant if and only if $\alpha x=\beta x=x$. Hence, we have

$$
\left(H^{*} B A\right)^{W(A)}=R \otimes M_{1,1} .
$$

Remark 4.4 Our computation of the ring of invariants of polynomial tensor exterior algebra in Proposition 4.3 is based on the computation of the ring of invariants of polynomial algebra and the assumption that the ring of invariants of polynomial algebra is also a polynomial algebra. In the case $A=E_{E_{8}}^{5 b}$, however, the Weyl group does not satisfy the condition we assume in this section and the ring of invariants of polynomial algebra is no longer a polynomial algebra. Hence, both Theorem 4.1 and Theorem 4.2 do not apply in this case.

\section{$5 \mathcal{O}_{n-1}\left(x_{i}\right)$ and $\mathcal{D}_{n-1}$}

In this section, we collect some facts, which we need in the proof of Theorem 4.1 and Theorem 4.2.

For $i=1, \ldots, n$, the element $\mathcal{O}_{n-1}\left(x_{i}\right)$ in $\mathbb{F}_{q}\left[x_{1}, \ldots, x_{n}\right]$ is defined to be

$$
\mathcal{O}_{n-1}\left(x_{i}\right)=\prod_{x \in \mathbb{F}_{q}\left\{x_{2}, \ldots, x_{n}\right\}}\left(x_{i}+x\right) .
$$

We also define $\mathcal{O}_{n-2}\left(x_{i}\right)$ in $\mathbb{F}_{q}\left[x_{1}, \ldots, x_{n-1}\right]$ by

$$
\mathcal{O}_{n-2}\left(x_{i}\right)=\prod_{x \in \mathbb{F}_{q}\left\{x_{2}, \ldots, x_{n-1}\right\}}\left(x_{i}+x\right)
$$

for $n \geq 3$ and by

$$
\mathcal{O}_{0}\left(x_{i}\right)=x_{i}
$$

for $n=2$.

Using the same argument as in the proof of Proposition 2.5, we can easily obtain the following proposition. 
Proposition 5.1 For $i=1, \ldots, n$, we may express $\mathcal{O}_{n-1}\left(x_{i}\right)$ and $\mathcal{O}_{n-2}\left(x_{i}\right)$ in terms of Dickson invariants as follows:

$$
\begin{array}{ll}
\mathcal{O}_{n-1}\left(x_{i}\right)=\sum_{j=0}^{n-1}(-1)^{n-1-j} c_{n-1, j}\left(x_{2}, \ldots, x_{n}\right) x_{i} q^{j} & \text { for } n \geq 2, \\
\mathcal{O}_{n-2}\left(x_{i}\right)=\sum_{j=0}^{n-2}(-1)^{n-2-j} c_{n-2, j}\left(x_{2}, \ldots, x_{n-1}\right) x_{i}^{q^{j}} & \text { for } n>2 .
\end{array}
$$

We need Proposition 5.2 and Proposition 5.3 below in the proof of Proposition 7.5.

Proposition 5.2 In $\mathbb{F}_{q}\left[x_{1}, \ldots, x_{n-1}\right]$, we have the following equality:

$$
e_{n-1}\left(x_{1}, \ldots, x_{n-1}\right)=\mathcal{O}_{n-2}\left(x_{1}\right) e_{n-2}\left(x_{2}, \ldots, x_{n-1}\right)
$$

for $n \geq 3$, and

$$
e_{1}\left(x_{1}\right)=x_{1}
$$

for $n=2$.

Proof For $n=2$, the proposition is obvious. For $n \geq 3$, by Proposition 2.4, Proposition 2.5 and Proposition 5.1, we have

$$
\begin{aligned}
& e_{n-1}\left(x_{1}, \ldots, x_{n-1}\right) \\
= & Q_{0} \ldots Q_{n-2} d x_{1} \ldots d x_{n-1} \\
= & \sum_{j=0}^{n-2}(-1)^{n-2-j}\left(Q_{0} \ldots \widehat{Q}_{j} \ldots Q_{n-2} d x_{2} \ldots d x_{n-1}\right) x_{1}^{q^{j}} \\
= & \sum_{j=0}^{n-2}(-1)^{n-2-j} e_{n-2}\left(x_{2}, \ldots, x_{n-1}\right) c_{n-2, j}\left(x_{2}, \ldots, x_{n-1}\right) x_{1}^{q^{j}} \\
= & e_{n-2}\left(x_{2}, \ldots, x_{n-1}\right) \mathcal{O}_{n-2}\left(x_{1}\right) .
\end{aligned}
$$

Proposition 5.3 The obvious projection

$$
\pi: \mathbb{F}_{q}\left[x_{1}, \ldots, x_{n}\right] \longrightarrow \mathbb{F}_{q}\left[x_{1}, \ldots, x_{n-1}\right]
$$

maps $\mathcal{O}_{n-1}\left(x_{1}\right), e_{n-1}\left(x_{2}, \ldots, x_{n}\right)$ to $\mathcal{O}_{n-2}\left(x_{1}\right)^{q}, 0$, respectively.

Proof Since $e_{n-1}\left(x_{2}, \ldots, x_{n}\right)$ is divisible by $x_{n}$, we have

$$
\pi\left(e_{n-1}\left(x_{2}, \ldots, x_{n}\right)\right)=0
$$


as in the proof of Proposition 2.9. For $n=2$, the equality

$$
\pi\left(\mathcal{O}_{1}\left(x_{1}\right)\right)=\mathcal{O}_{0}\left(x_{1}\right)^{q}=x_{1}^{q}
$$

is obvious. For $n \geq 3$, by Proposition 2.9, we have

$$
\pi\left(c_{n-1, j}\left(x_{2}, \ldots, x_{n}\right)\right)=c_{n-2, j-1}\left(x_{2}, \ldots, x_{n-1}\right)^{q}
$$

for $j=1, \ldots, n-1$. Hence, we have

$$
\begin{aligned}
\pi\left(\mathcal{O}_{n-1}\left(x_{1}\right)\right) & =\pi\left(\sum_{j=0}^{n-1}(-1)^{n-1-j} c_{n-1, j}\left(x_{2}, \ldots, x_{n}\right) x_{1}^{q^{j}}\right) \\
& =\sum_{j=1}^{n-1}(-1)^{n-1-j} c_{n-2, j-1}\left(x_{2}, \ldots, x_{n-1}\right)^{q} x_{1}^{q^{j}} \\
& =\mathcal{O}_{n-2}\left(x_{1}\right)^{q} .
\end{aligned}
$$

For $a$ in $P_{n} \otimes E_{n}$, let

$$
\mathcal{D}_{n-1}(a)=\sum_{j=0}^{n-1}(-1)^{n-1-j} c_{n-1, j}\left(x_{2}, \ldots, x_{n}\right) Q_{j} a .
$$

Then, $\mathcal{D}_{n-1}$ induces a $P_{n}$-linear homomorphism

$$
\mathcal{D}_{n-1}: P_{n} \otimes E_{n}^{r} \longrightarrow P_{n} \otimes E_{n}^{r-1},
$$

which extends naturally to

$$
\mathcal{D}_{n-1}: K_{n} \otimes E_{n}^{r} \rightarrow K_{n} \otimes E_{n}^{r-1} .
$$

Proposition 5.4 For $i=1, \ldots, n$, we have

$$
\mathcal{D}_{n-1}\left(d x_{i}\right)=\mathcal{O}_{n-1}\left(x_{i}\right) \text {. }
$$

In particular, for $i=2, \ldots, n$, we have $\mathcal{D}_{n-1}\left(d x_{i}\right)=0$.

Proof By Proposition 5.1, we have

$$
\begin{aligned}
\mathcal{D}_{n-1}\left(d x_{i}\right) & =\sum_{j=0}^{n-1}(-1)^{n-1-j} c_{n-1, j}\left(x_{2}, \ldots, x_{n}\right) Q_{j} d x_{i} \\
& =\sum_{j=0}^{n-1}(-1)^{n-1-j} c_{n-1, j}\left(x_{2}, \ldots, x_{n}\right) x_{i}^{q^{j}} \\
& =\mathcal{O}_{n-1}\left(x_{i}\right) .
\end{aligned}
$$

Geometry \& Topology Monographs 11 (2007) 
On the other hand, by the definition of $\mathcal{O}_{n-1}\left(x_{i}\right)$, we have

$$
\mathcal{O}_{n-1}\left(x_{i}\right)=0
$$

for $i=2, \ldots, n$. Hence, we have $\mathcal{D}_{n-1}\left(d x_{i}\right)=0$ for $i=2, \ldots, n$.

Let $g_{1}$ be an element in $G L_{n-1}\left(\mathbb{F}_{q}\right)$. We consider the following matrix

$$
\bar{g}_{1}=\left(\begin{array}{c|c}
1 & 0 \\
\hline 0 & g_{1}
\end{array}\right) .
$$

We need Proposition 5.5 and Proposition 5.6 below in the proof of Proposition 7.8.

Proposition 5.5 For $g_{1}$ in $G L_{n-1}\left(\mathbb{F}_{q}\right)$, there holds

$$
\mathcal{D}_{n-1}\left(\bar{g}_{1} a\right)=\bar{g}_{1} \mathcal{D}_{n-1}(a) \text {. }
$$

Proof Suppose that

$$
a=\sum_{i=1}^{n} a_{i} d x_{i},
$$

where $a_{i} \in K_{n}$ for $i=1, \ldots, n$. Since

$$
\bar{g}_{1} d x_{1}=d x_{1},
$$

and since, for $i=2, \ldots, n$,

$$
\bar{g}_{1} d x_{i}
$$

is in $E_{n-1}^{1}$, we have

$$
\bar{g}_{1} a=\left(\bar{g}_{1} a_{1}\right) d x_{1}+a_{2}^{\prime} d x_{2}+\cdots+a_{n}^{\prime} d x_{n}
$$

for some $a_{i}^{\prime} \in K_{n}$ for $i=2, \ldots, n$. Hence, by Proposition 5.4, we have

$$
\mathcal{D}_{n-1}\left(\bar{g}_{1} a\right)=\left(\bar{g}_{1} a_{1}\right) \mathcal{O}_{n-1}\left(x_{1}\right) .
$$

On the other hand, by Proposition 5.4, we have

$$
\bar{g}_{1} \mathcal{D}_{n-1}(a)=\bar{g}_{1}\left(a_{1} \mathcal{O}_{n-1}\left(x_{1}\right)\right)=\left(\bar{g}_{1} a_{1}\right) \mathcal{O}_{n-1}\left(x_{1}\right) .
$$

Let $\mathbb{P}^{n-2}$ be the projective space

$$
\left(\mathbb{F}_{q}^{n-1} \backslash\{(0, \ldots, 0)\}\right) / \sim,
$$

where $\ell \sim \ell^{\prime}$ if and only if there is $\alpha \in \mathbb{F}_{q}^{\times}$such that $\ell=\alpha \ell^{\prime}$. 
Proposition 5.6 If $a \in P_{n}$ is divisible by $\alpha_{2} x_{2}+\cdots+\alpha_{n} x_{n}$ for arbitrary $\left(\alpha_{2}, \ldots, \alpha_{n}\right)$ $\in \mathbb{P}^{n-2}$, then $a$ is divisible by $e_{n-1}\left(x_{2}, \ldots, x_{n}\right)$.

Proof Since the number of elements in $\mathbb{P}^{n-2}$ is equal to the homogeneous degree $1+q+\cdots+q^{n-2}$ of $e_{n-1}\left(x_{2}, \ldots, x_{n}\right)$, it suffices to show that $e_{n-1}\left(x_{2}, \ldots, x_{n}\right)$ is a product of elements of the form $\alpha_{2} x_{2}+\cdots+\alpha_{n} x_{n}$, where $\left(\alpha_{2}, \ldots, \alpha_{n}\right)$ ranges over $\mathbb{P}^{n-2}$. It is clear that $e_{n-1}\left(x_{2}, \ldots, x_{n}\right)$ is divisible by $x_{n}$. So, we have

$$
e_{n-1}\left(x_{2}, \ldots, x_{n}\right)=b x_{n}
$$

for some $b \in P_{n}$. It is also clear that $e_{n-1}\left(x_{2}, \ldots, x_{n}\right)$ is invariant under the action of $S L_{n-1}\left(\mathbb{F}_{q}\right)$. There is $g_{1} \in S L_{n-1}\left(\mathbb{F}_{q}\right)$ such that $\alpha_{2} x_{2}+\cdots+\alpha_{n} x_{n}=\bar{g}_{1} x_{n}$. Hence, on the one hand, we have

$$
\bar{g}_{1} e_{n-1}\left(x_{2}, \ldots, x_{n}\right)=\left(\bar{g}_{1} b\right)\left(\alpha_{2} x_{2}+\cdots+\alpha_{n} x_{n}\right)
$$

and, on the other hand, we have

$$
\bar{g}_{1} e_{n-1}\left(x_{2}, \ldots, x_{n}\right)=e_{n-1}\left(x_{2}, \ldots, x_{n}\right) .
$$

Therefore, $e_{n-1}\left(x_{2}, \ldots, x_{n}\right)$ is divisible by arbitrary $\alpha_{2} x_{2}+\cdots+\alpha_{n} x_{n}$. This completes the proof.

\section{$6 \quad$ Proof of Theorem 4.1}

In order to prove Theorem 4.1, we recall the strategy to compute rings of invariants given by Wilkerson in [9, Section 3]. It can be stated in the following form.

Theorem 6.1 Suppose that $G$ is a subgroup of $G L_{n}\left(\mathbb{F}_{q}\right)$ and $G$ acts on the polynomial algebra $\mathbb{F}_{q}\left[x_{1}, \ldots, x_{n}\right]$ in the obvious manner. Let $f_{1}, \ldots, f_{n}$ be homogeneous $G-$ invariant polynomials in $\mathbb{F}_{q}\left[x_{1}, \ldots, x_{n}\right]$. Let $R$ be the subalgebra of $\mathbb{F}_{q}\left[x_{1}, \ldots, x_{n}\right]$ generated by $f_{1}, \ldots, f_{n}$. Then, $R$ is a polynomial algebra $\mathbb{F}_{q}\left[f_{1}, \ldots, f_{n}\right]$ and the ring of invariants $\mathbb{F}_{q}\left[x_{1}, \ldots, x_{n}\right]^{G}$ is equal to the subalgebra $R$ if and only if $\mathbb{F}_{q}\left[x_{1}, \ldots, x_{n}\right]$ is integral over $R$ and $\operatorname{deg} f_{1} \ldots \operatorname{deg} f_{n}=|G|$.

In the statement of Theorem $6.1, \operatorname{deg} f$ is the homogeneous degree of $f$, that is, we define the degree $\operatorname{deg} x_{i}$ of indeterminate $x_{i}$ to be 1 . For the proof of this theorem, we refer the reader to Smith's book [8, Corollaries 2.3.2 and 5.5.4, and Proposition 5.5.5] and Wilkerson's paper [9, Section 3]. 
Proof of Theorem 4.1 As we mentioned, in order to prove Theorem 4.1, it suffices to show the following:

(1) homogeneous polynomials $\mathcal{O}_{n-1}\left(x_{1}\right), f_{2}, \ldots, f_{n}$ are $G$-invariant;

(2) indeterminates $x_{1}, \ldots, x_{n}$ are integral over $R$;

(3) the product of homogeneous degrees of $\mathcal{O}_{n-1}\left(x_{1}\right), f_{2}, \ldots, f_{n}$ is equal to the order of $G$, that is,

$$
\operatorname{deg} \mathcal{O}_{n-1}\left(x_{1}\right) \operatorname{deg} f_{2} \ldots \operatorname{deg} f_{n}=|G| .
$$

By definition, $f_{2}, \ldots, f_{n}$ are $G_{1}$-invariant, and so they are also $G$-invariant. It follows from Theorem 6.1 that $x_{2}, \ldots, x_{n}$ are integral over $R_{1}=\mathbb{F}_{q}\left[f_{2}, \ldots, f_{n}\right]$, and so they are integral over $R$. It is also immediate from Theorem 6.1 that $\operatorname{deg} f_{2} \ldots \operatorname{deg} f_{n}=\left|G_{1}\right|$. It is clear from the definition of $\mathcal{O}_{n-1}\left(x_{1}\right)$ that $\operatorname{deg} \mathcal{O}_{n-1}=2^{n-1}$. Hence, we have

$$
\operatorname{deg} \mathcal{O}_{n-1}\left(x_{1}\right) \operatorname{deg} f_{2} \ldots \operatorname{deg} f_{n}=2^{n-1}\left|G_{1}\right|=|G| .
$$

So, it remains to show the following:

(1) $\mathcal{O}_{n-1}\left(x_{1}\right)$ is $G$-invariant and

(2) $x_{1}$ is integral over $R$.

First, we deal with (1). By the definition of $\mathcal{O}_{n-1}\left(x_{1}\right)$, we have that

$$
g \mathcal{O}_{n-1}\left(x_{1}\right)
$$

is a product of

$$
g\left(x_{1}+x\right)=x_{1}+\sum_{j=2}^{n} a_{1, j}\left(g^{-1}\right) x_{j}+g x,
$$

where $x$ ranges over $\mathbb{F}_{q}\left\{x_{2}, \ldots, x_{n}\right\}$. As $x$ ranges over $\mathbb{F}_{q}\left\{x_{2}, \ldots, x_{n}\right\}$, the sum

$$
\sum_{j=2}^{n} a_{1, j}\left(g^{-1}\right) x_{j}+g x
$$

also ranges over $\mathbb{F}_{q}\left\{x_{2}, \ldots, x_{n}\right\}$. Hence, we have

$$
g \mathcal{O}_{n-1}\left(x_{1}\right)=\mathcal{O}_{n-1}\left(x_{1}\right) \text {. }
$$

Next, we deal with (2). By Proposition 5.1, we have

$$
\mathcal{O}_{n-1}(X)=X^{q^{n-1}}+\sum_{j=0}^{n-2}(-1)^{n-1-j} c_{n-1, j}\left(x_{2}, \ldots, x_{n}\right) X^{q^{j}} .
$$


Since Dickson invariants $c_{n-1, j}\left(x_{2}, \ldots, x_{n}\right)$ are in $R_{1}=\mathbb{F}_{2}\left[x_{2}, \ldots, x_{n}\right]^{G_{1}}$, the polynomial

$$
\varphi(X)=\mathcal{O}_{n-1}(X)-\mathcal{O}_{n-1}\left(x_{1}\right)
$$

is a monic polynomial in $R[X]$. It is clear that

$$
\varphi\left(x_{1}\right)=0 .
$$

Hence, the indeterminate $x_{1}$ is integral over $R$. This completes the proof.

\section{Proof of Theorem 4.2}

Let $G_{0}$ be the subgroup of $G$ consisting of the following matrices:

$$
\left(\begin{array}{c|c}
1 & m \\
\hline 0 & 1_{n-1}
\end{array}\right),
$$

where $m \in M_{1, n-1}\left(\mathbb{F}_{q}\right), 1_{n-1}$ is the identity matrix in $G L_{n-1}\left(\mathbb{F}_{q}\right)$. Let $B_{n}$ be the set of subsets of

$$
\{2, \ldots, n\} \text {. }
$$

Let $B_{n, r}$ be the subset of $B_{n}$ such that $J \in B_{n, r}$ if and only if

$$
J=\left\{j_{1}, \ldots, j_{r}\right\} \quad \text { and } \quad 1<j_{1}<\cdots<j_{r} \leq n .
$$

We write $d x_{J}$ for

$$
d x_{j_{1}} \ldots d x_{j_{r}}
$$

and we define $d x_{\emptyset}$ to be 1 .

The following proposition is nothing but the particular case of Theorem 4.1 and Theorem 4.2.

Proposition 7.1 The ring of invariants $P_{n}^{G_{0}}$ is given as follows:

$$
P_{n}^{G_{0}}=\mathbb{F}_{q}\left[\mathcal{O}_{n-1}\left(x_{1}\right), x_{2}, \ldots, x_{n}\right] .
$$

The ring of invariants $\left(P_{n} \otimes E_{n}\right)^{G_{0}}$ is a free $P_{n}^{G_{0}}$-module with the basis

$$
\left\{Q_{I} d x_{1} \ldots d x_{n}, d x_{J}\right\}
$$

where $I$ ranges over $A_{n-1}$ and $J$ ranges over $B_{n}$.

Now, we consider a $K_{n}$-basis for $K_{n} \otimes E_{n}$. 
Proposition 7.2 The elements

$$
Q_{0} \ldots Q_{n-2} d x_{1} \ldots d x_{n}, d x_{2}, \ldots, d x_{n}
$$

form a $K_{n}$-basis for $K_{n} \otimes E_{n}^{1}$.

Proof For dimensional reasons, it suffices to show that the above elements are linearly independent in $K_{n} \otimes E_{n}^{1}$. Suppose that

$$
a_{1} Q_{0} \ldots Q_{n-2} d x_{1} \ldots d x_{n}+a_{2} d x_{2}+\cdots+a_{n} d x_{n}=0
$$

in $K_{n} \otimes E_{n}^{1}$, where $a_{1}, \ldots, a_{n}$ are in $K_{n}$. Then, since

$$
Q_{0} \ldots Q_{n-2} d x_{1} \ldots d x_{n}=\sum_{i=1}^{n}(-1)^{n-i} e_{n-1}\left(x_{1}, \ldots, \widehat{x}_{i}, \ldots, x_{n}\right) d x_{i}
$$

we have

$$
\begin{aligned}
(-1)^{n-1} a_{1} e_{n-1}\left(\widehat{x}_{1}, \ldots, x_{n}\right) & =0, \\
a_{2}+(-1)^{n-2} a_{1} e_{n-1}\left(x_{1}, \widehat{x}_{2}, \ldots, x_{n}\right) & =0, \\
& \vdots \\
a_{n}+(-1)^{0} a_{1} e_{n-1}\left(x_{1}, \ldots, x_{n-1}, \widehat{x}_{n}\right) & =0 .
\end{aligned}
$$

Thus, solving this linear system, we obtain $a_{1}=0, \ldots, a_{n}=0$.

Proposition 7.3 The elements

$$
Q_{I} d x_{1} \ldots d x_{n}, d x_{J}
$$

form a $K_{n}$-basis for $K_{n} \otimes E_{n}^{r}$, where $I \in A_{n-1, n-r}$ and $J \in B_{n, r}$.

Proof Again, for dimensional reasons, it suffices to show that the above elements are linearly independent in $K_{n} \otimes E_{n}^{r}$. Suppose that

$$
\sum_{I \in A_{n-1, n-r}} a_{I} Q_{I} d x_{1} \ldots d x_{n}+\sum_{J \in B_{n, r}} b_{J} d x_{J}=0,
$$

where $a_{I}, b_{J}$ are in $K_{n}$. The linear independence of the terms $d x_{J}$ is clear. Hence, it remains to show that $a_{I}=0$ for each $I$.

Fix $I \in A_{n-1, n-r}$ and let $K=S_{n-1} \backslash I$. Then, applying $Q_{K}$ to the both sides of the above equality, we have

$$
\operatorname{sign}(K, I) a_{I} Q_{0} \ldots Q_{n-2} d x_{1} \ldots d x_{n}+\alpha=0
$$

in $K_{n} \otimes E_{n}^{1}$, where $\alpha$ is a linear combination of $d x_{2}, \ldots, d x_{n}$ over $K_{n}$. Hence, by Proposition 7.2, we have $a_{I}=0$ for each $I \in A_{n-1, n-r}$. 
Suppose that $a$ is in $P_{n} \otimes E_{n}^{1}$. Then, on the one hand, we may express $a$ as follows:

$$
a=\varphi_{1} d x_{1}+\cdots+\varphi_{n} d x_{n}
$$

where $\varphi_{1}, \ldots, \varphi_{n}$ are in $P_{n}$. On the other hand, by Proposition 7.2, we may express $a$ as follows:

$$
a=a_{1} Q_{0} \ldots Q_{n-2} d x_{1} \ldots d x_{n}+a_{2} d x_{2}+\cdots+a_{n} d x_{n},
$$

where $a_{1}, \ldots, a_{n}$ are in $K_{n}$. Observe that terms $a$ and $\varphi$ are unique in the above expressions.

We need to show that $a_{1}, \ldots, a_{n}$ are in $P_{n}$ if $a$ is $G_{0}$-invariant.

Proposition 7.4 There are polynomials $a_{i}^{\prime}$ over $\mathbb{F}_{q}$ in $n$ variables such that

$$
a_{i} e_{n-1}\left(x_{2}, \ldots, x_{n}\right)=a_{i}^{\prime}\left(x_{1}, \ldots, x_{n}\right)
$$

for $i=1, \ldots, n$.

Proof For $i=1$, we apply $\mathcal{D}_{n-1}$ to $a$. Then, we have

$$
\mathcal{D}_{n-1}(a)=\varphi_{1} \mathcal{O}_{n-1}\left(x_{1}\right)
$$

On the other hand, we have

$$
\begin{aligned}
\mathcal{D}_{n-1}(a) & =(-1)^{n-1} a_{1} Q_{0} \ldots Q_{n-1} d x_{1} \ldots d x_{n} \\
& =(-1)^{n-1} a_{1} \mathcal{O}_{n-1}\left(x_{1}\right) e_{n-1}\left(x_{2}, \ldots, x_{n}\right) .
\end{aligned}
$$

Hence, we obtain

$$
a_{1}=\left(e_{n-1}\left(x_{2}, \ldots, x_{n}\right)\right)^{-1} \varphi_{1}
$$

and

$$
a_{1}^{\prime}\left(x_{1}, \ldots, x_{n}\right)=\varphi_{1} .
$$

For $i=2, \ldots, n$, applying $Q_{0}, \ldots, Q_{n-2}$ to $a$, we have a linear system

$$
\begin{aligned}
Q_{0} a & =a_{2} x_{2}+\cdots+a_{n} x_{n}, \\
Q_{1} a & =a_{2} x_{2}^{q}+\cdots+a_{n} x_{n}^{q}, \\
& \vdots \\
Q_{n-2} a & =a_{2} x_{2}^{q^{n-2}}+\cdots+a_{n} x_{n}^{q^{n-2}} .
\end{aligned}
$$


Writing this linear system in terms of matrix, we have

$$
\left(\begin{array}{c}
Q_{0} a \\
Q_{1} a \\
\vdots \\
Q_{n-2} a
\end{array}\right)=A\left(\begin{array}{c}
a_{2} \\
a_{3} \\
\vdots \\
a_{n}
\end{array}\right),
$$

where

$$
A=\left(\begin{array}{ccc}
x_{2} & \ldots & x_{n} \\
x_{2}^{q} & \ldots & x_{n}^{q} \\
\vdots & \ddots & \vdots \\
x_{2}^{q^{n-2}} & \ldots & x_{n}^{q^{n-2}}
\end{array}\right)
$$

It is clear that $\operatorname{det} A=e_{n-1}\left(x_{2}, \ldots, x_{n}\right) \neq 0$. It is also clear that each entry of $A$ is in $P_{n-1}$. Therefore, for some $\varphi_{i, j}$ in $P_{n-1}$, we have

$$
a_{i}=e_{n-1}\left(x_{2}, \ldots, x_{n}\right)^{-1}\left(\sum_{j=1}^{n} \varphi_{i, j} Q_{j} a\right) .
$$

Since $Q_{j} a$ is in $P_{n}$, by letting

$$
a_{i}^{\prime}\left(x_{1}, \ldots, x_{n}\right)=\sum_{j=1}^{n} \varphi_{i, j} Q_{j} a,
$$

we obtain the required results.

Proposition 7.5 Suppose that $a$ is $G_{0}$-invariant. Then $a_{i}^{\prime}\left(x_{1}, \ldots, x_{n}\right)$ in Proposition 7.4 are also $G_{0}$-invariant for $i=1, \ldots, n$.

Proof For $g \in G_{0}$, we have

$$
g\left(d x_{i}\right)=d x_{i}
$$

for $i=2, \ldots, n$ and, since $G_{0} \subset S L_{n}\left(\mathbb{F}_{q}\right)$, we have

$$
g\left(d x_{1} \ldots d x_{n}\right)=d x_{1} \ldots d x_{n} .
$$

Since the action of Milnor operations $Q_{j}$ commutes with the action of the general linear group $G L_{n}\left(\mathbb{F}_{q}\right)$, we have

$$
g\left(Q_{0} \ldots Q_{n-2} d x_{1} \ldots d x_{n}\right)=Q_{0} \ldots Q_{n-2} d x_{1} \ldots d x_{n} .
$$


Hence, we have

$$
g a=\left(g a_{1}\right) Q_{0} \ldots Q_{n-2} d x_{1} \ldots d x_{n}+\left(g a_{2}\right) d x_{2}+\cdots+\left(g a_{n}\right) d x_{n} .
$$

Thus, if $g a=a$, we have $g a_{1}=a_{1}, \ldots, g a_{n}=a_{n}$. It is also clear that $e_{n-1}\left(x_{2}, \ldots, x_{n}\right)$ is $G_{0}$-invariant. Hence, $a_{i}^{\prime}\left(x_{1}, \ldots, x_{n}\right)$ are also $G_{0}$-invariant for $i=1, \ldots, n$.

Proposition 7.6 Suppose that $a$ is $G_{0}$-invariant. Then, $a_{1}^{\prime}\left(x_{1}, \ldots x_{n}\right)$ is divisible by $x_{n}$.

Proof Let us consider the coefficient $\varphi_{n}$ of $d x_{n}$; we have

$$
\varphi_{n} e_{n-1}\left(x_{2}, \ldots, x_{n}\right)=a_{n}^{\prime}\left(x_{1}, \ldots, x_{n}\right)+a_{1}^{\prime}\left(x_{1}, \ldots, x_{n}\right) e_{n-1}\left(x_{1}, \ldots, x_{n-1}\right) .
$$

Since $a_{1}^{\prime}\left(x_{1}, \ldots, x_{n}\right)$ and $a_{n}^{\prime}\left(x_{1}, \ldots, x_{n}\right)$ are $G_{0}$-invariant, there are polynomials $a_{1}^{\prime \prime}, a_{n}^{\prime \prime}$ over $\mathbb{F}_{q}$ in $n$ variables such that

$$
\begin{aligned}
& a_{1}^{\prime}\left(x_{1}, \ldots, x_{n}\right)=a_{1}^{\prime \prime}\left(\mathcal{O}_{n-1}\left(x_{1}\right), x_{2}, \ldots, x_{n}\right), \\
& a_{n}^{\prime}\left(x_{1}, \ldots, x_{n}\right)=a_{n}^{\prime \prime}\left(\mathcal{O}_{n-1}\left(x_{1}\right), x_{2}, \ldots, x_{n}\right) .
\end{aligned}
$$

Since $\mathcal{O}_{n-1}\left(x_{1}\right), x_{2}, \ldots, x_{n}$ are algebraically independent, it suffices to show that

$$
a_{1}^{\prime \prime}\left(y_{1}, x_{2}, \ldots, x_{n-1}, 0\right)=0
$$

for algebraically independent $y_{1}, x_{2}, \ldots, x_{n-1}$.

Substituting $x_{n}=0$, we have the obvious projection

$$
\pi: \mathbb{F}_{q}\left[x_{1}, \ldots, x_{n}\right] \rightarrow \mathbb{F}_{q}\left[x_{1}, \ldots, x_{n-1}\right] .
$$

It is clear from Proposition 5.2 and Proposition 5.3 that

$$
\begin{array}{rlr}
\pi\left(e_{n-1}\left(x_{1}, \ldots, x_{n-1}\right)\right) & =e_{n-1}\left(x_{1}, \ldots, x_{n-1}\right) & \\
& =\mathcal{O}_{n-2}\left(x_{1}\right) e_{n-2}\left(x_{2}, \ldots, x_{n-1}\right) & \text { for } n \geq 3, \\
\pi\left(e_{1}\left(x_{1}\right)\right) & =\mathcal{O}_{0}\left(x_{1}\right) & \\
\pi\left(e_{n-1}\left(x_{2}, \ldots, x_{n}\right)\right) & =0, & \\
\pi\left(\mathcal{O}_{n-1}\left(x_{1}\right)\right) & =\mathcal{O}_{n-2}\left(x_{1}\right)^{q} . &
\end{array}
$$

Hence, for $n \geq 3$, we have

$$
0=a_{n}^{\prime \prime}\left(y^{q}, x_{2}, \ldots, x_{n-1}, 0\right)+y a_{1}^{\prime \prime}\left(y^{q}, x_{2}, \ldots, x_{n-1}, 0\right) e_{n-2}\left(x_{2}, \ldots, x_{n-1}\right),
$$

where $y=\mathcal{O}_{n-2}\left(x_{1}\right)$ and $y, x_{2}, \ldots, x_{n-1}$ are algebraically independent. Applying the partial derivative $\partial / \partial y$, we have

$$
a_{1}^{\prime \prime}\left(y^{q}, x_{2}, \ldots, x_{n-1}, 0\right) e_{n-2}\left(x_{2}, \ldots, x_{n-1}\right)=0 .
$$


Hence, we have

$$
a_{1}^{\prime \prime}\left(y^{q}, x_{2}, \ldots, x_{n-1}, 0\right)=0 .
$$

Since $y^{q}, x_{2}, \ldots, x_{n-1}$ are algebraically independent, we have the required result.

For $n=2$, we have

$$
0=a_{2}^{\prime \prime}\left(y^{q}, 0\right)+y a_{1}^{\prime \prime}\left(y^{q}, 0\right) .
$$

Applying the partial derivative $\partial / \partial y$, we have

$$
a_{1}^{\prime \prime}\left(y^{q}, 0\right)=0
$$

and the required result.

Lemma 7.7 Suppose that $g_{1} \in G L_{n-1}\left(\mathbb{F}_{q}\right)$ and that $a$ is $G_{0}$-invariant. Then, $\bar{g}_{1} a$ is also $G_{0}$-invariant.

Proof Suppose that for each $g$, there is a $g^{\prime} \in G_{0}$ such that $g \bar{g}_{1}=\bar{g}_{1} g^{\prime}$. If it is true, then for any $G_{0}$-invariant $a$, we have

$$
g \bar{g}_{1} a=\bar{g}_{1} g^{\prime} a=g_{1} a .
$$

Hence, $g_{1}$ induces a homomorphism from $P_{n}^{G_{0}}$ to $P_{n}^{G_{0}}$. So, it suffices to show that for each $g$ in $G_{0}$, there is a $g^{\prime} \in G_{0}$ such that $g g_{1}=g_{1} g^{\prime}$, which is immediate from the following equality:

$$
\left(\begin{array}{c|c}
1 & m \\
\hline 0 & 1_{n-1}
\end{array}\right)\left(\begin{array}{c|c}
1 & 0 \\
\hline 0 & g_{1}
\end{array}\right)=\left(\begin{array}{c|c}
1 & 0 \\
\hline 0 & g_{1}
\end{array}\right)\left(\begin{array}{c|c}
1 & m g_{1} \\
\hline 0 & 1_{n-1}
\end{array}\right),
$$

where $m \in M_{1, n-1}\left(\mathbb{F}_{q}\right)$ and $1_{n-1}$ stands for the identity matrix in $G L_{n-1}\left(\mathbb{F}_{q}\right)$.

Proposition 7.8 Suppose that $a$ is $G_{0}$-invariant. Then, $a_{1}, \ldots, a_{n}$ are in $P_{n}$.

Proof Firstly, we verify that $a_{1}$ is in $P_{n}$. To this end, we prove that the element $a_{1}^{\prime}\left(x_{1}, \ldots, x_{n}\right)$ is divisible by $e_{n-1}\left(x_{2}, \ldots, x_{n}\right)$. Let $\ell=\alpha_{2} x_{2}+\cdots+\alpha_{n} x_{n}$, where $\alpha_{2}, \ldots, \alpha_{n} \in \mathbb{F}_{q}$ and $\ell \neq 0$. By Proposition 5.6, it suffices to show that $a_{1}^{\prime}\left(x_{1}, \ldots, x_{n}\right)$ is divisible by $\ell$. There is $g_{1}$ in $G L_{n-1}\left(\mathbb{F}_{q}\right)$ such that $\bar{g}_{1}\left(x_{n}\right)=\ell$. Since, by Lemma 7.7, $\bar{g}_{1}^{-1} a$ is also in $\left(P_{n} \otimes E_{n}^{1}\right)^{G_{0}}$, there is an element $f$ in $P_{n}$ such that

$$
\mathcal{D}_{n-1}\left(\bar{g}_{1}^{-1} a\right)=f x_{n} \mathcal{O}_{n-1}\left(x_{1}\right) \text {. }
$$


Here we have

$$
\mathcal{O}_{n-1}\left(x_{1}\right) a_{1}^{\prime}\left(x_{1}, \ldots, x_{n}\right)=\mathcal{D}_{n-1}(a)=\bar{g}_{1} \mathcal{D}_{n-1}\left(\bar{g}_{1}^{-1} a\right)=\mathcal{O}_{n-1}\left(x_{1}\right) \bar{g}_{1}(f) \ell .
$$

So we have

$$
a_{1}^{\prime}\left(x_{1}, \ldots, x_{n}\right)=\left(\bar{g}_{1} f\right) \ell
$$

Secondly, we verify that $a_{i}$ are in $P_{n}$ for $i=2, \ldots, n$, which follows from the fact that

$$
\varphi_{i}=a_{i}+(-1)^{n-i} a_{1} e_{n-1}\left(x_{1}, \ldots, \widehat{x}_{i}, \ldots, x_{n}\right) .
$$

Proof of Proposition 7.1 Suppose that $a$ is in $P_{n} \otimes E_{n}^{r}$ and $G_{0}$-invariant. By Proposition 7.3, there are $a_{I}, b_{J} \in K_{n}$ such that

$$
a=\sum_{I \in A_{n-1, n-r}} a_{I} Q_{I} d x_{1} \ldots d x_{n}+\sum_{J \in B_{n, r}} b_{J} d x_{J}
$$

It suffices to show that $a_{I}, b_{J}$ are in $P_{n}$.

Firstly, we verify that $a_{I}$ is in $P_{n}$. Choose $I$ and let $K=S_{n-1} \backslash I$. Then, we have

$$
Q_{K} a=\operatorname{sign}(K, I) a_{I} Q_{0} \ldots Q_{n-2} d x_{1} \ldots d x_{n}+\sum_{J \in B_{n, r}} b_{J} Q_{K} d x_{J}
$$

By Proposition 7.8, $\operatorname{sign}(K, I) a_{I}$ is in $P_{n}$ and, by definition, $\operatorname{sign}(K, I) \neq 0$, hence $a_{I}$ is also in $P_{n}$.

Secondly, we prove that $b_{J}$ is in $P_{n}$. Put

$$
a^{\prime}=a-\sum_{I \in A_{n-1, n-r}} a_{I} Q_{I} d x_{1} \ldots d x_{n}=\sum_{J \in B_{n, r}} b_{J} d x_{J} .
$$

It is clear that $a^{\prime}$ is also in $P_{n} \otimes E_{n}^{r}$. Hence, $b_{J}$ is in $P_{n}$. This completes the proof.

Now, we complete the proof of Theorem 4.2.

Proof of Theorem 4.2 Suppose that $a$ is an element in $P_{n} \otimes E_{n}$ and that $a$ is also $G$-invariant. It suffices to show that $a$ is a linear combination of $\left\{v_{i}, Q_{I} d x_{1} \ldots, d x_{n}\right\}$ over $P_{n}^{G}$. It is clear that $a$ is also $G_{0}$-invariant. Hence, by Proposition 7.1, there are $a_{I}$, $b_{J}$ in $P_{n}^{G_{0}}=\mathbb{F}_{q}\left[\mathcal{O}_{n-1}\left(x_{1}\right), x_{2} \ldots, x_{n}\right]$ such that

$$
a=\sum_{I} a_{I} Q_{I} d x_{1} \ldots d x_{n}+\sum_{J} b_{J} d x_{J} .
$$


Thus, we have

$$
a=\sum_{I} \sum_{k \geq 0} a_{I, k} \mathcal{O}_{n-1}\left(x_{1}\right)^{k} Q_{I} d x_{1} \ldots d x_{n}+\sum_{J} \sum_{k \geq 0} b_{J, k} \mathcal{O}_{n-1}\left(x_{1}\right)^{k} d x_{J},
$$

where $a_{I, k}, b_{J, k}$ are in $P_{n-1}=\mathbb{F}_{q}\left[x_{2}, \ldots, x_{n}\right]$. Since, by Theorem 4.1, $g \in G$ acts trivially on $\mathcal{O}_{n-1}\left(x_{1}\right)$, and since $g \in G \subset S L_{n}\left(\mathbb{F}_{q}\right)$ acts trivially on $Q_{I} d x_{1} \ldots d x_{n}$, we have

$$
g a=\sum_{k \geq 0} \sum_{I}\left(g a_{I, k}\right) \mathcal{O}_{n-1}\left(x_{1}\right)^{k} Q_{I} d x_{1} \ldots d x_{n}+\sum_{k \geq 0} \sum_{J}\left(g b_{J, k}\right) \mathcal{O}_{n-1}\left(x_{1}\right)^{k}\left(g d x_{J}\right) .
$$

It is clear that $g d x_{J}$ is in $E_{n-1}$. As a $P_{n-1}$-module, $\left(P_{n} \otimes E_{n}^{r}\right)^{G_{0}}$ is a free $P_{n-1}$-module with the basis

$$
\left\{\mathcal{O}_{n-1}\left(x_{1}\right)^{k} d x_{J}, \mathcal{O}_{n-1}\left(x_{1}\right)^{k} Q_{I} d x_{1} \ldots d x_{n}\right\}
$$

Hence, we have

$$
g\left(a_{I, k}\right)=a_{I, k} .
$$

Thus, $a_{I, k}$ is in $P_{n-1}^{G_{1}}$ and so $a_{I}$ is in $P_{n}^{G}$. Put

$$
a^{\prime}=a-\sum_{I} a_{I} Q_{I} d x_{1} \ldots d x_{n}
$$

Then, $a^{\prime}$ is also in the ring of invariants $\left(P_{n} \otimes E_{n}\right)^{G}$, and we have

$$
a^{\prime}=\sum_{k \geq 0}\left(\sum_{J} b_{J, k} d x_{J}\right) \mathcal{O}_{n-1}\left(x_{1}\right)^{k} .
$$

Hence, $\sum_{J} b_{J, k} d x_{J}$ is in the ring of invariants $\left(P_{n-1} \otimes E_{n-1}\right)^{G_{1}}$. By the assumption on the ring of invariants $\left(P_{n-1} \otimes E_{n-1}\right)^{G_{1}}$, there are polynomials $b_{1, k}, \ldots, b_{2^{n-1}, k}$ in $P_{n-1}^{G_{1}}$ such that

$$
\sum_{J} b_{J, k} d x_{J}=\sum_{i=1}^{2^{n-1}} b_{i, k} v_{i}
$$

Thus, writing $b_{i}$ for $\sum_{k \geq 0} b_{i, k} \mathcal{O}_{n-1}\left(x_{1}\right)^{k}$, we have

$$
a=\sum_{i=1}^{2^{n-1}} b_{i} v_{i}+\sum_{I} a_{I} Q_{I} d x_{1} \ldots d x_{n}
$$

where $b_{i}, a_{I}$ are in $P_{n}^{G}$. This completes the proof. 


\section{A Appendix}

In [7], Mùi used the determinant of the $k \times k$ matrix $\left(x_{j}^{q^{i} \ell}\right)$ whose $(\ell, j)$ entry is $x_{j}^{q^{i} \ell}$ to describe the Dickson invariant $\left[i_{1}, \ldots, i_{k}\right]$. Using these Dickson invariants, he defined the Mùi invariant $\left[r: i_{1}, \ldots, i_{n-r}\right]$. In this appendix, we verify in Proposition A.4 that the Mùi invariant

$$
Q_{i_{1}} \ldots Q_{i_{n-r}} d x_{1} \ldots d x_{n}
$$

in this paper is indeed equal to the Mùi invariant $\left[r: i_{1}, \ldots, i_{n-r}\right]$, up to sign.

Firstly, we recall the definitions. See [7, Section 2] for the definition of $\left[i_{1}, \ldots, i_{k}\right]$ and and [7, Defintion 4.3] for the definition of $\left[r: i_{1}, \ldots, i_{n-r}\right]$.

Definition A.1 The Dickson invariant $\left[i_{1}, \ldots, i_{k}\right]\left(x_{1}, \ldots, x_{k}\right) \in \mathbb{F}_{q}\left[x_{1}, \ldots, x_{k}\right]$ is defined by

$$
\sum_{\sigma} \operatorname{sgn}(\sigma) x_{\sigma(1)}^{q^{i_{1}}} \ldots x_{\sigma(k)}^{q^{i_{k}}}=\operatorname{det}\left(x_{j}^{q^{i_{\ell}}}\right),
$$

where $\sigma$ ranges over the set of permutations of $\{1, \ldots, k\}$ and $\operatorname{sgn}(\sigma)$ is the sign of the permutation $\sigma$.

Definition A.2 The Mùi invariant $\left[r: i_{1}, \ldots, i_{n-r}\right] \in P_{n} \otimes E_{n}^{r}$ is defined by

$$
\left[r: i_{1}, \ldots, i_{n-r}\right]=\sum_{J} \operatorname{sgn}\left(\sigma_{J}\right) d x_{j_{1}} \ldots d x_{j_{r}}\left[i_{1}, \ldots, i_{n-r}\right]\left(x_{j_{r+1}}, \ldots, x_{j_{n}}\right),
$$

where

$$
\sigma_{J}=\left(\begin{array}{ccc}
1, & \ldots, & n \\
j_{1}, & \ldots, & j_{n}
\end{array}\right)
$$

ranges over the set of permutations of $\{1, \ldots, n\}$ such that $j_{1}<\cdots<j_{r}$ and $j_{r+1}<\cdots<j_{n}$. The above $\sigma_{J}$ corresponds to the subset $J=\left\{j_{1}, \ldots, j_{r}\right\}$ of order $r$ of $\{1, \ldots, n\}$.

Secondly, we prove the following proposition.

Proposition A.3 There holds

$$
\begin{aligned}
{\left[i_{1}, \ldots, i_{k}\right]\left(x_{1}, \ldots, x_{k}\right) } & =Q_{i_{k}} \ldots Q_{i_{1}} d x_{1} \ldots d x_{k} \\
& =(-1)^{k(k-1) / 2} Q_{i_{1}} \ldots Q_{i_{k}} d x_{1} \ldots d x_{k} .
\end{aligned}
$$


Proof We prove the first equality in this proposition by induction on $k$. Indeed, in the case $k=1$, the proposition holds. Suppose that $k \geq 2$ and that there holds the equality

$$
\left[i_{2}, \ldots, i_{k}\right]\left(x_{1}, \ldots, x_{k-1}\right)=Q_{i_{k}} \ldots Q_{i_{2}} d x_{1} \ldots d x_{k-1} .
$$

Using the cofactor expansion (or the Laplace development) of the $k \times k$ matrix $\left(x_{j}^{q^{i} \ell}\right)$ along the first row

$$
\left(x_{1}^{q_{1}^{i_{1}}}, x_{2}^{q_{1}^{i_{1}}}, \ldots, x_{k}^{q^{i_{1}}}\right)
$$

we have

$$
\begin{aligned}
{\left[i_{1}, \ldots, i_{k}\right]\left(x_{1}, \ldots, x_{k}\right) } & =\sum_{s=1}^{k}(-1)^{s+1}\left[i_{2}, \ldots, i_{k}\right]\left(x_{1}, \ldots, \widehat{x}_{s}, \ldots, x_{k}\right) x_{s}^{q_{1}} \\
& =\sum_{s=1}^{k}(-1)^{s+1}\left(Q_{i_{k}} \ldots Q_{i_{2}} d x_{1} \ldots \widehat{d x_{s}} \ldots d x_{k}\right) x_{s}^{q^{i_{1}}} \\
& =Q_{i_{k}} \ldots Q_{i_{2}}\left(\sum_{s=1}^{k}(-1)^{s+1} x_{s}^{q_{1}^{i_{1}}} d x_{1} \ldots \widehat{d x_{s}} \ldots d x_{k}\right) \\
& =Q_{i_{k}} \ldots Q_{i_{1}} d x_{1} \ldots d x_{k} .
\end{aligned}
$$

So, the first equality holds. The second equality is immediate from the fact that

$$
Q_{i_{k}} \ldots Q_{i_{1}}=(-1)^{k(k-1) / 2} Q_{i_{1}} \ldots Q_{i_{k}} .
$$

Finally, we state and prove the following proposition.

Proposition A.4 There holds

$$
\begin{aligned}
{\left[r: i_{1}, \ldots, i_{n-r}\right] } & =(-1)^{(n-r) r} Q_{i_{n-r}} \ldots Q_{i_{1}} d x_{1} \ldots d x_{n} \\
& =(-1)^{(n-r) r+(n-r)(n-r-1) / 2} Q_{i_{1}} \ldots Q_{i_{n-r}} d x_{1} \ldots d x_{n}
\end{aligned}
$$

Proof As in the definition of $\left[r: i_{1}, \ldots, i_{n-r}\right]$, let $\sigma_{J}$ be a permutation of $\{1, \ldots, n\}$ with $\sigma_{J}(1)<\cdots<\sigma_{J}(r), \sigma_{J}(r+1)<\cdots<\sigma_{J}(n)$ and we denote by $j_{k}$ the value $\sigma_{J}(k)$ of $\sigma_{J}$ at $k$. Let $I(J)$ be the ideal of $P_{n} \otimes E_{n}$ generated by $d x_{j_{r+1}}, \ldots, d x_{j_{n}}$. Let

$$
p_{J}: P_{n} \otimes E_{n} \rightarrow P_{n} \otimes E_{n} / I(J)
$$

be the projection. It is clear that

$$
P_{n} \otimes E_{n}^{r} /\left(\left(P_{n} \otimes E_{n}^{r}\right) \cap I(J)\right)=P_{n}
$$


and for $f$ in $P_{n} \otimes E_{n}^{r}$, we have

$$
f=\sum_{J} p_{J}(f) d x_{j_{1}} \ldots d x_{j_{r}} .
$$

So, by Proposition A.3, in order to prove the proposition, it suffices to show that

$$
p_{J}\left(Q_{i_{n-r}} \ldots Q_{i_{1}} d x_{1} \ldots d x_{n}\right)=(-1)^{(n-r) r} \operatorname{sgn}\left(\sigma_{J}\right) Q_{i_{n-r}} \ldots Q_{i_{1}} d x_{j_{r+1}} \ldots d x_{j_{n}} .
$$

Suppose that

$$
\psi\left(Q_{i_{n-r}} \ldots Q_{i_{1}}\right)=1 \otimes Q_{i_{n-r}} \ldots Q_{i_{1}}+\sum a \otimes a^{\prime},
$$

where $\psi$ is the coproduct of the Steenrod algebra. We may choose $a^{\prime}$, so that $a^{\prime}=Q_{e_{1}} \ldots Q_{e_{\ell}}$ and $\ell<n-r$. Thus, $a^{\prime}\left(d x_{j_{r+1}} \ldots d x_{j_{n}}\right)$ belongs to $I(J)$.

Then, there holds

$$
\begin{aligned}
& Q_{i_{n-r}} \ldots Q_{i_{1}} d x_{1} \ldots d x_{n} \\
= & \operatorname{sgn}\left(\sigma_{J}\right) Q_{i_{n-r}} \ldots Q_{i_{1}} d x_{j_{1}} \ldots d x_{j_{n}} \\
= & (-1)^{(n-r) r} \operatorname{sgn}\left(\sigma_{J}\right) d x_{j_{1}} \ldots d x_{j_{r}} Q_{i_{n-r}} \ldots Q_{i_{1}} d x_{j_{r+1}} \ldots d x_{j_{n}} \\
& +\sum(-1)^{r \operatorname{deg} a^{\prime}} \operatorname{sgn}\left(\sigma_{J}\right)\left(a d x_{j_{1}} \ldots d x_{j_{r}}\right)\left(a^{\prime} d x_{j_{r+1}} \ldots d x_{j_{n}}\right) .
\end{aligned}
$$

Hence, we have

$$
p_{J}\left(Q_{i_{n-r}} \ldots Q_{i_{1}} d x_{1} \ldots d x_{n}\right)=(-1)^{(n-r) r} \operatorname{sgn}\left(\sigma_{J}\right) Q_{i_{n-r}} \ldots Q_{i_{1}} d x_{j_{r+1}} \ldots d x_{j_{n}}
$$

as required.

\section{References}

[1] K Andersen, J Grodal, J Møller, A Viruel, The classification of p-compact groups for $p$ odd arXiv:math.AT/0302346

[2] D J Benson, Polynomial invariants of finite groups, London Mathematical Society Lecture Note Series 190, Cambridge University Press, Cambridge (1993) MR1249931

[3] M C Crabb, Dickson-Mùi invariants, Bull. London Math. Soc. 37 (2005) 846-856 MR2186717

[4] LE Dickson, A fundamental system of invariants of the general modular linear group with a solution of the form problem, Trans. Amer. Math. Soc. 12 (1911) 75-98 MR1500882

[5] R L Griess, Jr, Elementary abelian p-subgroups of algebraic groups, Geom. Dedicata 39 (1991) 253-305 MR1123145

Geometry \& Topology Monographs 11 (2007) 
[6] M Kameko, M Mimura, On the Rothenberg-Steenrod spectral sequence for the mod 3 cohomology of the classifying space of the exceptional Lie group $E_{8}$, from: "Proceedings of the Nishida Fest (Kinosaki 2003)", Geom. Topol. Monogr. 10 209-222

[7] H Mùi, Modular invariant theory and cohomology algebras of symmetric groups, J. Fac. Sci. Univ. Tokyo Sect. IA Math. 22 (1975) 319-369 MR0422451

[8] L Smith, Polynomial invariants of finite groups, Research Notes in Mathematics 6, A K Peters Ltd., Wellesley, MA (1995) MR1328644

[9] C Wilkerson, A primer on the Dickson invariants, from: "Proceedings of the Northwestern Homotopy Theory Conference (Evanston, Ill., 1982)", Contemp. Math. 19, Amer. Math. Soc., Providence, RI (1983) 421-434 MR711066 A corrected version is available at the Hopf Topology Archive.

Department of Mathematics, Faculty of Regional Science

Toyama University of International Studies, 65-1 Higashikuromaki, Toyama, 930-1292, Japan

Department of Mathematics, Faculty of Science, Okayama University

3-1-1 Tsushima-naka, Okayama, 700-8530, Japan

kameko@tuins.ac.jp, mimura@math.okayama-u.ac.jp

Received: 26 Feb 2005 Revised: 12 Sept 2005 